TRANSACTIONS OF THE

Volume 276, Number 1, March 1983

\title{
ON THE GRÖSSENCHARACTER OF AN ABELIAN VARIETY IN A PARAMETRIZED FAMILY \\ BY
}

ROBERT S. RUMELY

\begin{abstract}
We consider families of abelian varieties parametrized by classical theta-functions, and show that specifying the family and a CM point in Siegel space determines the grössencharacter of the corresponding $\mathrm{CM}$ abelian variety. We associate an adelic group to the family, and describe the kernel of the grössencharacter as the pull-back of the group under the map in Shimura's Reciprocity Law.
\end{abstract}

The purpose of this paper is to give a formula for the grössencharacter of an abelian variety with complex multiplication, belonging to a family parametrized over the moduli space by theta-functions. In a previous paper [7] we have given a special case of the formula for elliptic curves, focusing on applications. Here we present the general case.

It has long been known that the grössencharacter determines the zeta-function of the variety (Deuring [3] for elliptic curves; Shimura and Taniyama [16], with later contributions by Serre and Tate [17], for higher-dimensional abelian varieties). The grössencharacter controls other arithmetic properties of the variety as well $[17,15]$. However, it has been difficult to approach directly. The Jacobians of Fermat Curves (implicitly, in Weil [21]) are one class where it can be independently studied. This paper provides another class.

We study families $\mathscr{F}$ of abelian varieties, parametrized over Siegel space by maps $F(u, z): \mathbf{C}^{n} \times \mathfrak{Q}_{n} \rightarrow \mathbf{P}^{m}$ constructed from classical theta-functions. Arithmetic information about $F(u, z)$ provides arithmetic information about the abelian varieties. Each variety $\mathscr{F}(z)$ is isomorphic to a complex torus $\mathbf{C}^{n} / L(z)$ with $L(z) \subset(z \mathbf{1}) \mathbf{Q}^{2 n}$, so if $z$ is a $\mathrm{CM}$ point, the corresponding variety has complex multiplication. The grössencharacter indirectly comes from the functional equation of theta-functions under the symplectic group.

Our approach is based on the complementary relationship between abelian variety and modular functions, and uses Shimura's reciprocity laws for modular functions and theta-functions. We associate a certain adelic group to the family (its "projective group"), and describe the kernel of the grössencharacter as the pull-back of the group under the reciprocity law map. Thus, the data

$$
\text { Family + CM point in moduli space }
$$

is enough to specify both variety and grössencharacter.

Received by the editors February 18, 1982.

1980 Mathematics Subject Classification. Primary 10D25, 14K22; Secondary 10D20, 14K25.

Key words and phrases. Abelian variety, theta-function, complex multiplication, grössencharacter. 
The paper is divided into three sections. In the first we review facts about modular functions and complex multiplication. In the second we prove our main theorem, and in the third we illustrate it for one-, two-, and $n$-dimensional families. As was noted in [7], our results apply to the classical Legendre, Jacobi, and Hesse curves

$$
\begin{gathered}
y^{2}=x(x-1)(x-\lambda), \\
y^{2}=\left(1-x^{2}\right)\left(1-k^{2} x^{2}\right), \\
x^{3}+y^{3}+1=3 \mu x y .
\end{gathered}
$$

We determine the projective groups of each of these families. In the case of abelian surfaces, we give a family parametrized by functions which generalize the Jacobian elliptic functions, determine its projective group, and work out some numerical examples. In the $n$-dimensional case we reformulate a result of Shimura to give a subgroup of the projective group for a family with arbitrary polarization type. Really, we have barely touched the higher-dimensional case; these results may perhaps serve as a guide to interesting families for study.

This is a revised version of the author's doctoral thesis under Professor Goro Shimura at Princeton University. The author is very grateful to Professor Shimura for his kindness and generous help.

Notations and conventions. $\mathbf{Z}, \mathbf{Q}, \mathbf{R}$ and $\mathbf{C}$ mean as usual the ring of rational integers, and fields of rational, real, and complex numbers respectively. $\mathbf{A}$ will be the ring of rational adeles, $\mathbf{A}_{f}$ its finite part, and $\mathbf{Z}_{f}=\prod_{p} \mathbf{Z}_{p}$ the closure of $\mathbf{Z}$ in $\mathbf{A}_{f} . x_{\infty}$ will be the archimedean component of an adele $x ; x_{p}$ its component at the prime $p$. Similar notations will apply to other adelic objects.

Throughout the paper, $n$ is reserved for the dimension of the abelian varieties under consideration. All vectors will be column vectors. The transpose of a matrix $x$ is written ${ }^{t} x$, and 1 will denote the appropriate-dimensional identity matrix. $Y^{\times}$ means the group of invertible elements of a ring $Y$.

All number fields are taken to be subfields of $\mathbf{C}$. If $K$ is a number field, $K_{\mathrm{ab}}$ denotes the maximal abelian extension of $K$, and $K_{\mathrm{A}}^{\times}$the idele group of $K$; we regard $K^{\times}$as embedded in $K_{\mathbf{A}}^{\times}$. The Artin map will be written $[s, K]$ for $s \in K_{\mathbf{A}}^{\times}$.

1. Preliminaries on abelian varieties, modular functions, and theta-functions. Siegel's upper half-space $\mathfrak{E}_{n}$ is the set of complex symmetric matrices of degree $n$, with positive definite imaginary part. The symplectic group $\operatorname{Sp}(n, \mathbf{R})$ acts on $\mathfrak{S}_{n}$ by

$$
\gamma(z)=(a z+b)(c z+d)^{-1} \text { for } z \in \mathfrak{Q}_{n}, \gamma=\left(\begin{array}{ll}
a & b \\
c & d
\end{array}\right) \in \operatorname{Sp}(n, \mathbf{R}) \text {. }
$$

A Siegel modular form of weight $k(k \in \mathbf{Z})$ is a holomorphic function $f(z)$ on $\mathfrak{S}_{n}$ (finite at the cusps, if $n=1$ ), satisfying

$$
f(z)=\left(\left.f\right|_{k} \gamma\right)(z)=\operatorname{det}(c z+d)^{-k} f(\gamma(z))
$$

for all $\gamma$ in some congruence subgroup $\Gamma(N)$ of $\operatorname{Sp}(n, \mathbf{Z})$. Such a function has a Fourier expansion of the type

$$
f(z)=\sum_{\substack{i \alpha=\alpha \\ \alpha \in M_{n}(\mathbf{Z})}} c_{\alpha} e(\operatorname{tr}(\alpha z) / N) \quad\left(e(w)=e^{2 \pi i w}\right)
$$


The arithmetic modular forms are those with cyclotomic Fourier coefficients. One defines the space $\mathcal{Q}_{k}\left(\mathbf{Q}_{\mathrm{ab}}\right)$ of arithmetic automorphic forms of weight $k$ to be the set of quotients $g(z) / h(z)$, where $g(z)$ and $0 \neq h(z)$ are arithmetic modular forms of weights $k+m$ and $m$ respectively, with arbitrary $m$.

Let $J$ be the $2 n \times 2 n$ matrix

$$
J=\left(\begin{array}{cc}
0 & -1 \\
1 & 0
\end{array}\right)
$$

and define the groups of restricted symplectic similitudes

$$
\begin{aligned}
& G_{\mathbf{A}^{+}}=\left\{\left.\gamma \in \mathrm{GL}_{2 n}(\mathbf{A})\right|^{t} \gamma J \gamma=\nu(\gamma) J, \text { where } \nu(\gamma) \in \mathbf{A}^{\times} \text {and } \nu(\gamma)_{\infty}>0\right\}, \\
& G_{\mathbf{Q}^{+}}=G_{\mathbf{A}^{+}} \cap \mathrm{GL}_{2 n}(\mathbf{Q}) \text {. }
\end{aligned}
$$

There is an associative right action of $G_{\mathbf{A}^{+}}$on the $\mathbb{Q}_{k}\left(\mathbf{Q}_{\mathrm{ab}}\right)$, written $f \rightarrow f^{u}$ for $f \in \mathbb{Q}_{\boldsymbol{k}}\left(\mathbf{Q}_{\mathrm{ab}}\right)$ and $u \in G_{\mathbf{A}^{+}}$, characterized by the properties (see [12])

I. $f^{\gamma}=\left.f\right|_{k} \gamma=\operatorname{det}(c z+d)^{-k} f(\gamma(z))$ for $\gamma=\left(\begin{array}{cc}a & b \\ c & d\end{array}\right) \in G_{\mathbf{Q}^{+}}$(note that the factor $\nu(\gamma)^{k / 2}$ one frequently sees is omitted here).

II. $f^{\iota(t)}=f^{[t, \mathbf{Q}]}$ with

$$
\iota(t)=\left[\begin{array}{cc}
1 & 0 \\
0 & t^{-1} \mathbf{1}
\end{array}\right] \quad \text { for } t \in \mathbf{Z}_{f}^{\times},
$$

where for a modular form, $f^{[t, \mathbf{Q}]}$ means the modular form obtained by letting $[t, \mathbf{Q}]$ act on the Fourier coefficients.

III. The subgroup of $G_{\mathbf{A}^{+}}$fixing $f$ is open.

The quotient of $\mathfrak{S}_{n}$ by an appropriate congruence subgroup of $\operatorname{Sp}(n, \mathbf{Z})$ is the moduli space for abelian varieties with given polarization type. To construct the point corresponding to an abelian variety $A$ with a nondegenerate polarization $P$, take an isomorphism $\lambda$ from a complex torus $C^{n} / L$ onto $A$, and let $E(u, v)$ be the alternating Riemann form corresponding to a basic polar divisor in $P$. If $P$ determines the matrix of elementary divisors $\varepsilon=\operatorname{diag}\left(\varepsilon_{1}, \varepsilon_{2}, \ldots, \varepsilon_{n}\right)$, where $\varepsilon_{1}=1$, $\varepsilon_{i} \mid \varepsilon_{i+1}$ and $0<\varepsilon_{i} \in \mathbf{Z}$, then there is an $n \times 2 n$ complex matrix $\Omega$ (which we decompose as $\Omega=\left(\omega_{1} \omega_{2}\right)$ with square matrices $\left.\omega_{1}, \omega_{2}\right)$ whose columns form a basis for $\mathbf{Q} L$, such that

$$
\begin{aligned}
E(\Omega x, \Omega y) & ={ }^{t} x J y \text { for } x, y \in \mathbf{R}^{2 n}, \\
L & =\Omega\left(\begin{array}{ll}
1 & 0 \\
0 & \varepsilon
\end{array}\right) \mathbf{Z}^{2 n} .
\end{aligned}
$$

The point corresponding to $(A, P)$ is $z_{0}=\omega_{2}^{-1} \omega_{1} ; z_{0}$ is unique up to translation by

$$
\operatorname{Sp}(n, \mathbf{Z}) \cap\left(\begin{array}{ll}
1 & 0 \\
0 & \varepsilon
\end{array}\right)^{-1} \mathrm{GL}_{2 n}(\mathbf{Z})\left(\begin{array}{ll}
1 & 0 \\
0 & \varepsilon
\end{array}\right)
$$

Assume from now on that $A$ has many complex mutiplications, that is, $A$ is isogenous to a product $\Pi A_{i}^{r_{i}}$ of simple abelian varieties $A_{i}$, where each $\operatorname{End}\left(A_{i}\right) \otimes \mathbf{Q}$ is a CM field $K_{i}$ of degree $2 \cdot \operatorname{dim}\left(A_{i}\right)$. Fix an anti-isomorphism

$$
\tau: Y=M_{r_{1}}\left(K_{1}\right) \oplus \cdots \oplus M_{r_{d}}\left(K_{d}\right) \rightarrow \operatorname{End}(A) \otimes \mathbf{Q} .
$$


(The choice of an anti-isomorphism rather than an isomorphism is made for compatibility with Shimura's Reciprocity Law.) Then the formula

$$
\tau(y) \lambda(\Omega x)=\lambda\left(\Omega^{t} \xi(y) x\right) \text { for } x \in \mathbf{R}^{2 n}, y \in Y,
$$

determines a representation $\xi$ of $Y$ in $M_{2 n}(\mathbf{Q})$. There is an involution $\delta: Y \rightarrow Y$ such that for $u, v \in \mathbf{R}^{2 n}, y \in Y$,

$$
E\left(\Omega^{t} \xi(y) u, \Omega v\right)=E\left(\Omega u, \Omega^{t} \xi\left(y^{\delta}\right) v\right)
$$

$\delta$ coincides with complex conjugation on each of the fields $K_{i}$.

$\tau$ also determines an anti-representation $\phi$ of $Y$ in $M_{n}(\mathbf{C})$ via the complex structure provided by $\lambda$. Write $K$ for the center of $Y$, and $e_{i}$ for the identity element of $M_{r_{i}}\left(K_{i}\right)$, so $K=\bigoplus K_{i} e_{i}$. The restriction of $\phi$ to $K_{i}$ is equivalent to $r_{i}$ copies of a representation $\phi_{i}$, where the pair $\left(K_{i}, \phi_{i}\right)$ is a CM type in the sense of [16, p. 44]: when diagonalized, $\phi_{i}$ is the direct sum of embeddings $\sigma_{1}, \ldots, \sigma_{n_{i}}$ of $K_{i}$ into $\mathrm{C}$, such that from each pair of conjugate embeddings $\sigma$ and $\bar{\sigma}$, precisely one appears among the $\sigma_{i}$. Let $\left(K_{i}^{\prime}, \phi_{i}^{\prime}\right)$ be the reflex to $\left(K_{i}, \phi_{i}\right)$ (called the dual in [16, p. 70]), and let $K^{\prime}$ be the composite of the $K_{i}^{\prime}: K^{\prime}$ is a field, even though $K$ is in general not. There is a homomorphism

$$
\eta\left(k^{\prime}\right)=\sum_{i=1}^{d} \operatorname{det} \phi_{i}^{\prime}\left(N_{K^{\prime} / K_{i}^{\prime}}\left(k^{\prime}\right)\right) e_{i} .
$$

Since $\delta$ is the complex conjugation on $K_{i}, \eta\left(k^{\prime}\right) \cdot \eta\left(k^{\prime}\right)^{\delta} \in \mathbf{Q}$.

It is not hard to see that for any $y \in Y^{\times}$satisfying $y \cdot y^{\delta} \in \mathbf{Q}, \xi(y)$ belongs to $G_{\mathbf{Q}^{+}}$, and

$$
\xi(y)\left(z_{0}\right)=z_{0} .
$$

For this reason $\xi$ is called the embedding at the fixed point $z_{0} . \xi \circ \eta$ takes $K^{\prime \times}$ to $G_{\mathbf{Q}^{+}}$, and $K_{\mathbf{A}}^{\prime \times}$ to $G_{\mathbf{A}^{+}}$; it is the higher-dimensional "Reciprocity Law Map". Shimura's Reciprocity Law for Siegel modular functions [9, I, 2.7.3, II, 6.2.3] says that for any $f \in \mathbb{Q}_{0}\left(\mathbf{Q}_{\mathrm{ab}}\right)$ finite at $z_{0}, f\left(z_{0}\right)$ belongs to $K_{\mathrm{ab}}^{\prime}$; and for any $s \in K_{\mathrm{A}}^{\prime \times}$,

$$
f\left(z_{0}\right)^{\left[s, K^{\prime}\right]}=f^{\xi \circ \eta\left(s^{-1}\right)}\left(z_{0}\right) .
$$

Shimura's Reciprocity Law corresponds to the Fundamental Theorem of Complex Multiplication in the theory of abelian varieties. The following version of the Fundamental Theorem may be found in [10, Proposition 2.3]; see also [9, I, 4.3].

Proposition 1. Let $(A, P)$ be a polarized abelian variety with many complex multiplications, with isomorphisms $\tau: Y \rightarrow \operatorname{End}(A) \otimes \mathbf{Q}, \lambda: \mathbf{C}^{n} / L \rightarrow A$, and a Riemann form $E(u, v)$ as above. For any automorphism of $\mathbf{C}$ over $K^{\prime}$, let $s \in K_{\mathrm{A}}^{\prime \times}$ be such that $\sigma \mid K_{\mathrm{ab}}^{\prime}=\left[s, K^{\prime}\right]$. Then there is an isomorphism $\lambda^{\prime}: \mathrm{C}^{n} / \eta(s)^{-1} L \rightarrow A^{\sigma}$ such that $\lambda(v)^{\sigma}=\lambda^{\prime}\left(\eta(s)^{-1} v\right)$ for all $v \in \mathbf{Q} L / L$, and $P^{\sigma}$ determines the Riemann form $N \cdot E(u, v)$, where $N$ is the absolute norm of the ideal of $K^{\prime}$ associated to $s$.

If $\mathbf{k}$ is a field of definition for $A$, denote by $Y_{\mathbf{k}}$ the subalgebra of all $y \in Y$ rational over $\mathbf{k}$ (more properly, such that for some $m \in \mathbf{Z}, \tau(m y) \in \operatorname{End}(A)$ is rational over 
k) and let $C_{\mathbf{k}}$ be the center of $Y_{\mathbf{k}}$. The following proposition asserts the existence of the grössencharacter of an abelian variety with many complex multiplications, assumptions being as in Proposition 1.

Proposition 2. Suppose $A$ and its polarization $P$ are rational over a number field $\mathbf{k}$, and there is a maximal commutative semisimple subalgebra $D$ of $Y$ contained in $Y_{\mathbf{k}}$. Then $K^{\prime} \subset \mathbf{k}$, and every torsion point of $A$ is rational over $\mathbf{k}_{\mathrm{ab}}$. Further

1. For each $x \in \mathbf{k}_{\mathbf{A}}^{\times}$, writing $\mu(x)=\eta\left(N_{\mathbf{k} / K^{\prime}}(x)\right)$, there is a unique element $\alpha(x)$ of $Y^{\times}$such that $\alpha(x) \mu(x)^{-1} L=L$ and, for all $v \in \mathbf{Q} L / L$,

$$
\lambda\left(\alpha(x) \mu(x)^{-1} v\right)=\lambda(v)^{[\mathbf{x}, \mathbf{k}]} .
$$

The elements $\alpha(x)$ generate $C_{\mathbf{k}}$, and $Y_{\mathbf{k}}$ is the commutor of $C_{\mathbf{k}}$ in $Y$.

2. The matrices $\phi\left(C_{\mathbf{k}}\right)$ can be simultaneously diagonalized, giving linear representations $\chi_{j}: C_{\mathbf{k}} \rightarrow \mathbf{C}, j=1, \ldots, n$. For each $j$, if $\chi_{j}$ is extended to a homomorphism $\left(C_{\mathbf{k}} \otimes \mathbf{R}\right) \rightarrow \mathbf{C}$, then

$$
\psi_{j}(x)=\chi_{j}\left(\frac{\alpha(x)}{\mu(x)_{\infty}}\right) \quad \text { for } x \in \mathbf{k}_{\mathbf{A}}^{\times}
$$

is a grössencharacter of $\mathbf{k}$; the 1-dimensional part of $\zeta(s, A / \mathbf{k})$ and $\prod_{j=1}^{n} L\left(s, \psi_{j}\right) L\left(s, \bar{\psi}_{j}\right)$ coincide up to finitely many Euler factors, with equality if $C_{\mathbf{k}}$ is a field.

Proof. This is a mild generalization of results in Shimura $[8, \S 7.8]$ to the case of nonsimple abelian varieties, based on the version of the Fundamental Theorem given above. The condition $K^{\delta}=K$ assumed in $[8, \S 7.8]$ is automatic, since $K$ is the center of $Y$; and the fact that $K^{\prime} \subset \mathbf{k}$ is noted in [9, I, 4.2.1].

All the assertions except those about the rationality of the $\alpha(x)$ are proved word-for-word as in [8,7.40-7.42], with the algebra $D$ replacing the field $K$ there.

As for the rationality, first note that $y \in Y$ commutes with all the $\alpha(x)$ iff $y \in Y_{\mathbf{k}}$. Indeed, for each $\sigma \in \operatorname{Aut}(C / \mathbf{k})$ choose $x \in \mathbf{k}_{\mathbf{A}}^{\times}$such that $\sigma \mid \mathbf{k}_{\mathrm{ab}}=[x, \mathbf{k}] ; \mu(x)$ lies in the center $K_{\mathrm{A}}$ of $Y_{\mathrm{A}}$. Hence for any $y$ which commutes with all the $\alpha(x)$, and for all $v \in \mathbf{Q} L / L$,

$$
\begin{aligned}
\tau(y)^{\sigma} \lambda\left(\alpha(x) \mu(x)^{-1} v\right) & =\tau(y)^{\sigma} \lambda(v)^{\sigma}=\lambda(y v)^{\sigma} \\
& =\lambda\left(\alpha(x) \mu(x)^{-1} y v\right)=\lambda\left(y \alpha(x) \mu(x)^{-1} v\right) \\
& =\tau(y) \lambda\left(\alpha(x) \mu(x)^{-1} v\right)
\end{aligned}
$$

so that $\tau(y)^{\sigma}=\tau(y)$; conversely, if $\tau(y)$ is fixed by $\operatorname{Aut}(C / \mathbf{k})$ the same circle of equalities shows that $y$ commutes with the $\alpha(x)$.

By the uniqueness of $\alpha(x)$, if $x=\kappa \in \mathbf{k}$, then $\alpha(x)=\mu(k)$. Now, by the same method as in [9, I, Proposition 1.9.2] it can be shown that $\mu\left(\mathbf{k}^{\times}\right)$generates $K$ over $\mathbf{Q}$. Hence, letting $C$ be the subalgebra of $Y$ generated by the $\alpha(x)$, we have shown that $K \subset C \subset C_{\mathbf{k}} \subset Y$ and $Y_{\mathbf{k}}$ is the commutor of $C$ in $Y$. It is well known that in this situation $C=C_{\mathbf{k}}$.

Classical theta-functions provide specific examples of projective embeddings of abelian varieties. Writing $e(w)$ for $e^{2 \pi i w}$, the classical theta-function $\theta(u, z ; r, s)$, 
with fixed $r, s \in \mathbf{R}^{n}$ and arbitrary $u \in \mathbf{C}^{n}, z \in \mathfrak{E}_{n}$, is defined by

$$
\theta(u, z ; r, s)=\sum_{m \in \mathbf{Z}^{n}} e\left(\frac{1}{2}^{t}(m+r) z(m+r)+{ }^{t}(m+r)(u+s)\right) .
$$

Let $\varepsilon$ be a given matrix of elementary divisors, and $w$ an integer $\geqslant 3$. Then, taking $g$ to be a set of representatives for $(w \varepsilon)^{-1} \mathbf{Z}^{n} / \mathbf{Z}^{n}$,

$$
\Theta_{\varepsilon, w}(u, z)=(\theta(w u, w z ; j, 0))_{j \in \mathcal{g}}
$$

embeds $\mathbf{C}^{n} /(z \varepsilon) \mathbf{Z}^{2 n}$ into projective space, and the polarization induced by hyperplane sections is of type $\varepsilon$ (cf. [10, p. 676]). Other examples will be given later.

Classical theta-functions satisfy many identities, of which we note only a few; [10] is a good reference for these and others. Let $\{m\}$ denote the diagonal vector of a square matrix $m$, and put

$$
\Gamma_{\theta}=\left\{\gamma=\left(\begin{array}{ll}
a & b \\
c & d
\end{array}\right) \in \operatorname{Sp}(n, \mathbf{Z}) \mid\left\{{ }^{t} a c\right\} \equiv\left\{{ }^{t} b d\right\} \equiv 0 \bmod 2 \mathbf{Z}^{n}\right\}
$$

Then

$$
\theta(u+z k+l, z ; r, s)=e\left(-\left(\frac{1}{2}^{t} k z k+{ }^{t} k u\right)+\left({ }^{t} r k-{ }^{t} s l\right)\right) \cdot \theta(u, z ; r, s)
$$

for $k, l \in \mathbf{Z}^{n}$.

$$
\begin{aligned}
\theta\left({ }^{t}(c z+d)^{-1} u, \gamma(z) ; r, s\right)= & \lambda_{\gamma} e\left(\frac{1}{2}\left({ }^{t} r s-{ }^{t} r^{\prime} s^{\prime}\right)\right) \operatorname{det}(c z+d)^{1 / 2} \\
& \cdot e\left(\frac{1}{2}{ }^{t} u(c z+d)^{-1} c u\right) \cdot \theta\left(u, z ; r^{\prime}, s^{\prime}\right)
\end{aligned}
$$

for $\gamma \in \Gamma_{\theta}$, where $\left(\begin{array}{l}r^{\prime} \\ s^{\prime}\end{array}\right)={ }^{t} \gamma\left(\begin{array}{l}r \\ s\end{array}\right)$, and $\lambda_{\gamma}$ is an 8th root of unity depending only on $\gamma$ and the choice of branch of square-root.

$$
\theta\left({ }^{t}(c z+d)^{-1} u, \gamma(z) ; r, s\right)=\zeta \operatorname{det}(c z+d)^{1 / 2} e\left(\frac{1}{2}^{t} u(c z+d)^{-1} c u\right) \theta\left(u, z ; r^{\prime \prime}, s^{\prime \prime}\right)
$$

for $\gamma \in \operatorname{Sp}(n, \mathbf{Z})$, where $\zeta$ is a constant of absolute value 1 depending on $r, s$ and $\gamma$, and

$$
\left(\begin{array}{l}
r^{\prime \prime} \\
s^{\prime \prime}
\end{array}\right)={ }^{t} \gamma\left(\begin{array}{l}
r \\
s
\end{array}\right)+\frac{1}{2}\left(\begin{array}{c}
\left\{{ }^{t} a c\right\} \\
\left\{{ }^{t} b d\right\}
\end{array}\right) .
$$

$$
\theta(u+z p+q, z ; r, s)=e\left(-\frac{1}{2}^{t} p z p-{ }^{t} p(u+q+s)\right) \cdot \theta(u, z ; r+p, s+q)
$$

for $p, q \in \mathbf{R}^{n}$.

$$
\boldsymbol{\theta}(u, z ; r+k, s+l)=e\left({ }^{t} r l\right) \theta(u, z ; r, s) \text { for } k, l \in \mathbf{Z}^{n} .
$$

Equation (4a) provides the connection between classical theta-functions and modular forms of half-integral weight: for $r, s \in(1 / m) \mathbf{Z}^{n}$ and $\gamma \in \Gamma\left(2 m^{2}\right)$, by (4a) and (6)

$$
\theta(0, \gamma(z) ; r, s)=\lambda_{\gamma} \operatorname{det}(c z+d)^{1 / 2} \theta(0, z ; r, s) .
$$

Arithmetic automorphic forms of weight $k / 2$ are defined to be elements of $\boldsymbol{\theta}(0, z ; 0,0)^{k} \mathbb{Q}_{0}\left(\mathbf{Q}_{\mathrm{ab}}\right)$, for $k \in \mathbf{Z}$. It can be shown that when $k / 2 \in \mathbf{Z}$, this definition coincides with the earlier one. 
Any theory of modular forms of half-integral weight must take into account the indeterminateness of $\operatorname{det}(c z+d)^{1 / 2}$. In [12], Shimura enlarged $G_{\mathbf{A}^{+}}$to a group $\mathcal{G}_{\mathbf{A}^{+}}$ modeled on Weil's metaplectic group, containing "all possible" holomorphic branches of $\operatorname{det}(c z+d)^{1 / 2}$. He defined an action of it on the $Q_{k / 2}\left(\mathbf{Q}_{\mathrm{ab}}\right)$ and distinguished subgroups $\mathcal{G}_{\mathbf{Q}^{+}}$and $\iota_{1}\left(\mathbf{Z}_{f}^{\times}\right)$in it, such that with respect to these groups, the analogues of properties (I)-(III) hold. We recall only briefly that $\mathcal{S}_{\mathbf{Q}^{+}}$may be identified with the set of all pairs $(\alpha, \psi(z))$ where $\alpha \in G_{\mathbf{Q}^{+}}$and $\psi(z)=\zeta \cdot \operatorname{det}(c z+d)^{1 / 2}$ with a root of unity $\zeta$ and a holomorphic branch of square-root; and that $\iota_{1}\left(\mathbf{Z}_{f}^{\times}\right)$acts as usual on the Fourier coefficients. There is a natural surjective map $\pi: \mathcal{G}_{\mathbf{A}^{+}} \rightarrow G_{\mathbf{A}^{+}}$ which is open and continuous; conversely, there is a natural lifting of $\Gamma_{\theta}$ to $\mathcal{G}_{\mathbf{Q}^{+}}$, defined by

$$
\alpha \rightarrow(\alpha, \theta(0, \alpha(z) ; 0,0) / \theta(0, z ; 0,0)) .
$$

A congruence subgroup of $\mathcal{S}_{\mathbf{Q}^{+}}$is one containing the lift of some $\Gamma(m)$, with finite index.

Shimura then studied functions $f(u, z)$ on $\mathbf{C}^{n} \times \mathfrak{Q}_{n}$ which generalize the classical theta-functions. Fix $k \in \mathbf{Z}, 0 \leqslant w \in \mathbf{Q}$, take $\gamma=(\alpha, \psi(z)) \in \mathcal{G}_{\mathbf{Q}^{+}}$, and put

$$
\left(\left.f\right|_{k / 2, w} \gamma\right)(u, z)=\psi(z)^{-k} e\left(-\frac{1}{2} \nu(\alpha) w^{t} u(c z+d)^{-1} c u\right) f\left({ }^{t}(c z+d)^{-1} u, \alpha(z)\right) .
$$

He defined an arithmetic theta-function of weight $(k / 2, w)$ to be a holomorphic function on $\mathbf{C}^{n} \times \mathfrak{Q}_{n}$ such that

$$
\left(\left.f\right|_{k / 2, w} \gamma\right)(u, z)=f(u, z)
$$

for all $\gamma$ in some congruence subgroup of $\mathcal{G}_{\mathbf{Q}^{+}}$,

$$
f(u+z a+b, z)=e\left(-w\left(\frac{1}{2}^{t} a z a+{ }^{t} a u\right)\right) f(u, z)
$$

for all $\left(\begin{array}{l}a \\ b\end{array}\right)$ in some lattice in $\mathbf{Q}^{2 n}$, and

$$
\text { the Fourier coefficients of } f(u, z) \text { belong to } \mathbf{Q}_{\mathrm{ab}} \text {. }
$$

(The existence of a Fourier expansion is implied by (7) and (8). If $n=1$, one must also assume that the $f_{*}\left(\Omega_{z} v, z\right)$ defined below are finite at the cusps.) The $\theta(w u, w z ; r, s)$ with $r, s \in \mathbf{Q}^{n}$ provide examples of arithmetic theta-functions of weight $\left(\frac{1}{2}, w\right)$; the Fourier expansion of a classical theta-function is essentially given by its defining series.

For such a function, put

$$
f_{*}(u, z)=e\left(\frac{1}{2} w^{t}(u-\bar{u})(z-\bar{z})^{-1} u\right) f(u, z)
$$

and write $\Omega_{z}$ for the matrix $\left(z \mathbf{1}_{n}\right)$. If $n>1$, it can be verified that for each $x \in \mathbf{Q}^{2 n}, f_{*}\left(\Omega_{z} x, z\right)$ is an arithmetic modular form in $\mathbb{Q}_{k / 2}\left(\mathbf{Q}_{\mathrm{ab}}\right)$. When $n=1$, this is also true, except one must assume the finiteness at the cusps. Further, whenever $x, y \in \mathbf{Q}^{2 n}$ approximate $v=\mathbf{A}_{f}^{2 n}$ closely enough, $f_{*}\left(\Omega_{z} x, z\right)=f_{*}\left(\Omega_{z} y, z\right)$. Consequently, for adelic $v, f_{*}\left(\Omega_{z} v, z\right)$ can be defined to be this common value.

There is an action of $\mathcal{G}_{\mathbf{A}^{+}}$on the arithmetic theta-functions such that $f^{\gamma}=\left.f\right|_{k / 2, w} \gamma$ for $\gamma \in \mathcal{G}_{\mathbf{Q}^{+}}, \iota_{1}\left(\mathbf{Z}_{f}^{*}\right)$ acts on the Fourier coefficients, and the subgroup fixing a given $f$ is open. This action is compatible with the action on the $\mathbb{Q}_{k / 2}\left(\mathbf{Q}_{\mathrm{ab}}\right)$ in the sense 
that the following "Generic Reciprocity Law for Theta-functions" holds [12, Theorem 3.10, p. 54]:

Proposition 3. Given an arithmetic theta-function $f(u, z)$, for any $y \in \mathcal{G}_{\mathbf{A}^{+}}$with $x=\pi(y) \in G_{\mathbf{A}^{+}}$, and any $v \in \mathbf{A}_{f}^{2 n}$,

$$
f_{*}\left(\Omega_{z} v, z\right)^{y}=\left(f^{y}\right)_{*}\left(\Omega_{z}{ }^{t} x v, z\right) .
$$

It will be convenient for us to broaden the above notion of an arithmetic theta-function slightly, allowing it to be meromorphic instead of holomorphic in the variable $z$ : we will call an arithmetic theta-function of weight $(k / 2, w)$ a function $g(z) f(u, z)$, with $g(z) \in \mathbb{Q}_{j / 2}\left(\mathbf{Q}_{\mathrm{ab}}\right)$ and $f(u, z)$ an arithmetic theta-function in Shimura's sense of weight $(h / 2, w)$, where $j+h=k$. Proposition 3 remains valid for these functions.

2. The main theorem. Let $f_{0}(u, z), \ldots, f_{m}(u, z)$ be arithmetic theta-functions of the same weight $(k / 2, w)$, and suppose the map

$$
F(u, z)=\left(f_{j}(u, z)\right)_{0 \leqslant j \leqslant m}
$$

parametrizes a family of abelian varieties $\mathscr{F}$ : that is, for all $z$ in an open subset of $\mathfrak{G}_{n}$, the map $F_{z}(u)=F(u, z): \mathbf{C}^{n} \rightarrow \mathbf{P}^{m}$ is a nonsingular projective embedding of a complex torus. Because of the functional equation (8) satisfied by arithmetic theta-functions, the kernel of $F_{z}$ is a lattice $L(z) \subset \Omega_{z} \mathbf{Q}^{2 n}$; in fact there will be a matrix $M \in \mathrm{GL}_{2 n}(\mathbf{Q})$ independent of $z$, such that $L(z)=\Omega_{z} M Z^{2 n}$. Hence hyperplane sections induce the same type of polarization in each member of the family, and if $z$ is a $\mathrm{CM}$ point in $\mathfrak{S}_{n}$, the corresponding abelian variety $\mathscr{F}(z)$ has many complex multiplications.

DEFINITION 1. Such a map $F$ is said to parametrize the family of abelian varieties $\mathscr{F}$ by arithmetic theta-functions.

Consider the subgroup $\mathcal{P}$ of all $\mathbf{p} \in \mathcal{G}_{\mathbf{A}^{+}}$which fix $F$, that is, for which $f_{j}^{\mathbf{p}}(u, z)=$ $f_{j}(u, z), 0 \leqslant j \leqslant m$. Since the subgroup fixing each $f_{j}(u, z)$ is open, $\mathcal{P}$ is open, and so is its canonical projection $P=\pi(\mathscr{P}) \subset G_{\mathbf{A}^{+}}$. We will shortly see that the finite part of $P$ is compact.

Definition 2. $P=\pi(\mathscr{P})$ is called the projective group of the family $\mathscr{F}$.

We now give the main theorem, determining a field of rationality for $\mathscr{F}(z)$ and deriving a formula for its grössencharacters, if it has many complex multiplications. Write $\mathfrak{f}_{P}$ for the subfield of $\mathfrak{t}=\mathbb{Q}_{0}\left(\mathbf{Q}_{\mathrm{ab}}\right)$ fixed by $P$, and let $\mathfrak{f}_{P}[z]$ (resp. $\mathfrak{f}[z]$ ) be the subfields of $\mathbf{C}$ obtained by evaluating the functions at $z$. The first part of the theorem is similar to [11, Theorem 1.1, p. 368].

THEOREM 1. Suppose $z$ is a point for which there is an abelian variety $\mathscr{F}(z)$ in the family parametrized by $F$, and take $\mathscr{F}(z)$ to be polarized by the hyperplane sections. Then

(1) $\mathscr{F}(z)$ and its origin are rational over $\mathfrak{f}_{P}[z]$, and the smallest field of rationality for all the torsion points on $\mathscr{F}(z)$ is $\mathfrak{f}[z]$. 
(2) If $z=z_{0}$ is a $\mathrm{CM}$ point, then every element of $\operatorname{End}\left(\mathscr{F}\left(z_{0}\right)\right)$ is rational over $\mathbf{k}=\mathfrak{f}_{P}\left[z_{0}\right] \cdot K^{\prime}$, which is the classfield to $K^{\prime \times} \cdot(\xi \circ \eta)^{-1}(P) \subset K_{\mathbf{A}}^{\prime \times} ;$ all torsion points of $\mathscr{F}\left(z_{0}\right)$ are rational over $K_{\mathrm{ab}}^{\prime}$; and the grössencharacters $\psi_{j}$ of $\mathscr{F}\left(z_{0}\right) / \mathbf{k}$ are given by

$$
\psi_{j}(x)=\chi_{j}\left(\left(\frac{\eta\left(k^{\prime}\right)}{\eta\left(N_{\mathbf{k} / K^{\prime}}(x)\right)}\right)_{\infty}\right), \quad x \in \mathbf{k}_{\mathbf{A}}^{\times}, 1 \leqslant j \leqslant n,
$$

where $N_{\mathbf{k} / K^{\prime}}(x)=k^{\prime} \cdot u$, with $k^{\prime} \in K^{\prime \times}$ and $u \in(\xi \circ \eta)^{-1}(P)$. $k^{\prime}$ is unique iff $n=1$.

PRoof. In the following, we use $\mathbf{z}$ to denote a free variable in $\mathfrak{G}_{n}$, and regard $z$ and $z_{0}$ as fixed. Let $F_{*}(u, \mathbf{z}): \mathbf{C}^{n} \times \mathfrak{S}_{n} \rightarrow \mathbf{P}^{m}$ be the map obtained from $F$ by applying the *-operator to the coordinates of $F$. Since these all have the same weight, $F_{*}(u, z)$ gives the same projective embedding as $F(u, z)$. Adelizing the functions $\left(f_{j}\right)_{*}$ as above, we obtain a homomorphism

$$
\begin{aligned}
F_{*}: \mathbf{A}_{f}^{2 n} & \rightarrow \mathscr{F}(z), \\
v & \mapsto\left(\left(f_{j}\right)_{*}\left(\Omega_{z} v, z\right)\right)_{0<j<m}
\end{aligned}
$$

parametrizing the torsion points of $\mathscr{F}(z)$. Now is the union of the fields of moduli for abelian varieties with level $N$ structure, for all $N$, so certainly any field of rationality for the torsion points of $\mathscr{F}(z)$ will contain $\mathfrak{f}[z]$. In our case, since the quotients of the $\left(f_{j}\right)_{*}\left(\Omega_{z} v, \mathbf{z}\right)$ belong to $f$, the torsion points are actually rational over $\mathfrak{f}[z]$. Hence $\mathscr{F}(z)$ itself is also rational over $\mathfrak{f}[z]$.

Given $x \in P$, let $\mathbf{x}$ be a lift of $x$ to $\mathscr{P}$. Then, for any $v \in \mathbf{A}_{f}^{2 n}$, and for any indices $i, j$ such that $f_{j}\left(\Omega_{z} v, z\right) \neq 0$,

$$
\begin{aligned}
{\left.\left[\frac{\left(f_{i}\right)_{*}\left(\Omega_{\mathbf{z}} v, \mathbf{z}\right)}{\left(f_{j}\right)_{*}\left(\Omega_{\mathbf{z}} v, \mathbf{z}\right)}\right]^{x}\right|_{z} } & =\left.\left[\frac{\left(f_{i}^{\mathbf{x}}\right)_{*}\left(\Omega_{\mathbf{z}}{ }^{t} x v, \mathbf{z}\right)}{\left(f_{j}^{\mathbf{x}}\right)_{*}\left(\Omega_{\mathbf{z}}{ }^{t} x v, \mathbf{z}\right)}\right]\right|_{z} \\
& =\left.\left[\frac{\left(f_{i}\right)_{*}\left(\Omega_{\mathbf{z}}{ }^{t} x v, \mathbf{z}\right)}{\left(f_{j}\right)_{*}\left(\Omega_{\mathbf{z}}{ }^{t} x v, \mathbf{z}\right)}\right]\right|_{z}
\end{aligned}
$$

by Proposition 3 and the definition of the projective group. We can write this as

$$
\left.F_{*}\left(\Omega_{\mathbf{z}} v, \mathbf{z}\right)^{x}\right|_{z}=F_{*}\left(\Omega_{z}{ }^{t} x v, z\right) \text { for } x \in P .
$$

First suppose $z$ is a generic point for $f$ in the sense of [8, p. 137]. By definition, all automorphisms of $\mathfrak{f}[z]$ over $\mathfrak{f}_{P}[z]$ are induced by elements of $P$, and by formula (11), $\mathscr{F}(z)$ and its origin are stable under $P, \operatorname{so~}_{P}[z]$ is a field of rationality for them. We also see that the finite part of $P$ is compact, since otherwise formula (11) would provide a torsion point of $\mathscr{F}(z)$ with infinitely many images under $\operatorname{Gal}\left(\mathfrak{f}[z] / \mathfrak{f}_{P}[z]\right)$.

Now again let $z$ be arbitrary. Since the finite part of $P$ is compact, $\mathfrak{f}^{\mathfrak{L}}[z]$ is galois over $\mathfrak{f}_{P}[z]$, and as in $\left[11\right.$, Theorem 1.1, p. 368] to each $\sigma \in \operatorname{Gal}\left(\mathfrak{f}[z] / \mathfrak{f}_{P}[z]\right)$ there corresponds an $x \in P$ such that $h(z)^{\sigma}=h^{x}(z)$ for all $h \in \mathfrak{H}^{\mathrm{f}}$ with $h(z)$ finite. Thus, as before, $\mathscr{F}(z)$ and its origin are rational over $\mathfrak{f}_{P}[z]$. 
Next, suppose $\mathscr{F}\left(z_{0}\right)$ has many complex multiplications. By Shimura's Reciprocity Law (see $[9, I, 2.5 .5]), \mathbf{k}=\mathfrak{f}_{P}[z] \cdot K^{\prime}$ is the classfield to $K^{\prime \times} \cdot(\xi \circ \eta)^{-1}(P)$. Furthermore $\mathfrak{f}\left[z_{0}\right] \subset K_{\mathrm{ab}}^{\prime}$, and $K_{\mathrm{A}}^{\prime \times}$ acts on it by the formula $h\left(z_{0}\right)^{\left[s, K^{\prime}\right]}=h^{(\xi \circ \eta)\left(s^{-1}\right)}\left(z_{0}\right)$. Consider an element $y \in Y$ with $\tau(y) \in \operatorname{End}\left(\mathscr{F}\left(z_{0}\right)\right)$. Given $\sigma \in \operatorname{Gal}\left(K_{\mathrm{ab}}^{\prime} / \mathbf{k}\right)$, choose $s \in(\xi \circ \eta)^{-1}(P)$ such that $\sigma=\left[s, K^{\prime}\right]$. By the definition of $\xi$, for all $v \in \mathbf{A}_{f}^{2 n}$,

$$
\tau(y)\left(F_{*}\left(\Omega_{z_{0}} v, z_{0}\right)\right)=F_{*}\left(\Omega_{z_{0}}{ }^{t} \xi(y) v, z_{0}\right) .
$$

Applying $\sigma$ to both sides, using Shimura's Reciprocity Law, formula (11), and the fact that $\eta(s)$ is in the center of $Y_{\mathrm{A}}$, we obtain

$$
\begin{aligned}
F_{*}\left(\Omega_{z_{0}}{ }^{t} \xi(y) v, z_{0}\right)^{\sigma} & =F_{*}\left(\Omega_{z_{0}}{ }^{t} \xi\left(\eta\left(s^{-1}\right)\right)^{t} \xi(y) v, z_{0}\right) \\
& =F_{*}\left(\Omega_{z_{0}}{ }^{t} \xi(y)^{t} \xi\left(\eta\left(s^{-1}\right)\right) v, z_{0}\right) \\
& =\tau(y)\left(F_{*}\left(\Omega_{z_{0}}{ }^{t} \xi\left(\eta\left(s^{-1}\right)\right) v, z_{0}\right)\right)
\end{aligned}
$$

while

$$
\tau(y)^{\sigma}\left(F_{*}\left(\Omega_{z_{0}} v, z_{0}\right)\right)^{\sigma}=\tau(y)^{\sigma}\left(F_{*}\left(\Omega_{z_{0}}{ }^{t} \xi\left(\eta\left(s^{-1}\right)\right) v, z_{0}\right)\right) .
$$

Thus $\tau(y)^{\sigma}=\tau(y)$.

Lastly, to determine the grössencharacter of $\mathscr{F}\left(z_{0}\right) / \mathbf{k}$, note that in terms of the map $F_{*}$, Proposition 2 asserts the existence of a unique $\alpha \in Y^{\times}$corresponding to each $x \in \mathbf{k}_{\mathbf{A}}^{\times}$such that the following diagram commutes:

$$
\begin{array}{rcc}
\mathbf{A}_{f}^{2 n} & \stackrel{F_{*}}{\rightarrow} & \mathscr{F}\left(z_{0}\right) \\
{ }^{\prime} \xi\left(\alpha \cdot \eta\left(N_{\mathbf{k} / K^{\prime}}(x)^{-1}\right)\right) \downarrow & & \downarrow[x, \mathbf{k}] \\
\mathbf{A}_{f}^{2 n} & \stackrel{F_{*}}{\rightarrow} & \mathscr{F}\left(z_{0}\right)
\end{array}
$$

In view of Proposition 2 and the fact just proved that $Y=Y_{\mathrm{k}}, \alpha$ belongs to the center $K$ of $Y$. Now fix $x \in \mathbf{k}_{\mathbf{A}}^{\times}$and decompose $N_{\mathbf{k} / K^{\prime}}(x)=k^{\prime} \cdot u$ as in the statement of the theorem. All torsion points of $\mathscr{F}\left(z_{0}\right)$ are rational over $\mathfrak{f}\left[z_{0}\right] \subset K_{\mathrm{ab}}^{\prime}$, and $[x, \mathbf{k}] \mid K_{\mathrm{ab}}^{\prime}=\left[u, K^{\prime}\right]$. Traversing the diagram in two ways, we find first

$$
F_{*}\left(\Omega_{z_{0}} v, z_{0}\right)^{[x, \mathbf{k}]}=F_{*}\left(\Omega_{z_{0}}{ }^{t} \xi\left(\alpha \cdot \eta\left(k^{\prime}\right)^{-1} \eta\left(u^{-1}\right)\right) v, z_{0}\right)
$$

but also

$$
F_{*}\left(\Omega_{z_{0}} v, z_{0}\right)^{\left[u, K^{\prime}\right]}=F_{*}\left(\Omega_{z_{0}}{ }^{t} \xi\left(\eta\left(u^{-1}\right)\right) v, z_{0}\right)
$$

by Shimura's Reciprocity Law and formula $(11)$, since $\xi(\eta(u)) \in P$. Since this holds for all $v \in \mathbf{A}_{f}^{2 n}$ and $\xi$ is an injection, $\alpha=\eta\left(k^{\prime}\right)$.

When $n=1$, we have $Y=K$, and the isomorphism $\tau: K \rightarrow \operatorname{End}\left(\mathscr{F}\left(z_{0}\right)\right) \otimes \mathbf{Q}$ can be choosen so that the CM type $(K, \phi)$ is $(K, \mathrm{id})$; in this case $\tau$ is said to be normalized. $K^{\prime}$ coincides with $K$, and $\eta$ is the identity map. Since $\xi$ is injective, $k^{\prime}$ is unique.

When $n \neq 1,(\xi \circ \eta)^{-1}(P)$ contains $N_{F^{\prime}}^{-1} / Q(1)$, where $F^{\prime}$ is the totally real subfield of $K^{\prime}$, so $k^{\prime}$ is not unique. 
In the special case $n=1$, with $\tau$ normalized, the embedding $\xi: K \rightarrow M_{2}(\mathbf{Q})$ at the fixed point $z_{0}$ is just the map $q_{z_{0}}$ defined by

$$
\kappa\left(\begin{array}{c}
z_{0} \\
1
\end{array}\right)=q_{z_{0}}(\kappa)\left(\begin{array}{c}
z_{0} \\
1
\end{array}\right), \quad \kappa \in K
$$

We isolate this special case as

THEOREM 2. Let $\mathcal{F}$ be a family of elliptic curves parametrized by arithmetic theta-functions. The curve $\mathscr{F}(z)$ corresponding to $z \in \mathfrak{G}$ has complex multiplication iff $z=z_{0}$ lies in a quadratic imaginary field $K$, and if $\mathscr{U}_{F} \subset \mathrm{GL}_{2}(\mathbf{A})_{+}$is the projective group of $\mathscr{F}$, then for every $\mathrm{CM}$ point $z_{0}$ where $\mathscr{F}\left(z_{0}\right)$ is defined,

(1) The classfield $\mathbf{k}$ to $K^{\times} \cdot q_{z_{0}}^{-1}\left(\mathcal{Q}_{F}\right)$ is a field of definition for $\mathscr{F}\left(z_{0}\right) ; \mathbf{k}=K \cdot \mathfrak{f}_{\mathcal{Q}_{F}}\left[z_{0}\right]$.

(2) For each $s \in \mathbf{k}_{\mathbf{A}}^{\times}$, there is a unique decomposition $N_{\mathbf{k} / K}(s)=k \cdot u$ with $k=k(s)$ $\in K^{\times}, u \in q_{z_{0}}^{-1}\left(\mathcal{Q}_{F}\right)$.

(3) The grössencharacter of $\mathscr{F}\left(z_{0}\right) / \mathbf{k}$ is given by

$$
\chi(s)=\left(\frac{k(s)}{N_{\mathbf{k} / K}(s)}\right)_{\infty} \text { for } s \in \mathbf{k}_{\mathbf{A}}^{\times} .
$$

3. Examples. In this section we will compute the projective groups of some specific families. The following corollary of the Strong Approximation Theorem is useful in this regard. Recall that for $x \in G_{\mathbf{A}^{+}}, \nu(x)$ is the multiplier such that ${ }^{t} x J x=\nu(x) J$; when $n=1, \nu(x)$ is just $\operatorname{det}(x)$.

Proposition 4. Let $V$ be an open subgroup of $G_{\mathbf{A}^{+}}$. Then $V$ is generated over any smaller open subgroup by elements of $G_{\mathbf{Q}^{+}} \cap V$ and a set of representatives for $\nu^{-1}(\nu(V))$. If $V \subset W$ are two open subgroups such that $G_{\mathbf{Q}^{+}} \cap V=G_{\mathbf{Q}^{+}} \cap W$ and $\nu(V)=\nu(W)$, then $V=W$.

Proof. The second assertion follows immediately from the first. Suppose, then, that $U$ is a given small open subgroup of $V$. Put $S=G_{\infty^{+}} \cdot\{x \in U \mid \nu(x)=1\}$; note $S \subset V$. By the Strong Approximation Theorem (as stated in [12, Lemma 1.1, p. 39]), $G_{\mathbf{A}^{+}}=G_{\mathbf{Q}^{+}} \cdot \imath\left(\mathbf{Z}_{f}^{\times}\right) \cdot S$. If $v \in V$ is given, let $r$ be the representative for $\nu^{-1}(\nu(v))$, and decompose $v \cdot r^{-1}=\gamma \cdot \iota(t) \cdot s$ with $\gamma \in G_{\mathbf{Q}^{+}}, t \in \mathbf{Z}_{f}^{\times}, s \in S$ as asserted. Applying $\nu$ to both sides, we find that

$$
\nu\left(\gamma^{-1}\right)=\nu(\iota(t) \cdot s) \in \mathbf{Q}^{\times} \cap\left(\mathbf{R}_{+} \times \mathbf{Z}_{f}^{\times}\right)=\{1\}
$$

so that $\nu(s)=1$ and $t=1$, and $\gamma=v \cdot r^{-1} \cdot s^{-1} \in G_{\mathbf{Q}^{+}} \cap V$.

In the following, we write $\mathscr{U}$ for the subgroup of $G_{\mathrm{A}^{+}}$

$$
\mathcal{Q}=G_{\mathbf{A}^{+}} \cap \mathrm{GL}_{2 n}(\mathbf{R}) \times \prod_{p} \mathrm{GL}_{2 n}\left(\mathbf{Z}_{p}\right),
$$

and $\mathcal{U}(N)$ for the group

$$
\mathcal{Q}(N)=\left\{u \in \mathcal{Q} \mid u_{p} \equiv 1 \bmod N \cdot M_{2}\left(\mathrm{Z}_{p}\right) \text { for all } p\right\} .
$$


A. OnE-Dimensional EXAmples. (1) The Legendre curve $y^{2}=x(x-1)(x-\lambda)$ and the Jacobi curve $y^{2}=\left(1-x^{2}\right)\left(1-\mathbf{k}^{2} x^{2}\right)$ are 2-isogenous over $\mathbf{Q}(\lambda)$ to a third curve, parametrized by the Jacobian elliptic functions

$$
g: \begin{cases}y^{2}=1-x^{2}, & x=s n\left(u, \mathbf{k}^{2}(z)\right), \\ w^{2}=1-\mathbf{k}^{2}(z) x^{2}, & y=c n\left(u, \mathbf{k}^{2}(z)\right), \\ w=d n\left(u, \mathbf{k}^{2}(z)\right) .\end{cases}
$$

Here $\lambda(z)=\mathbf{k}^{2}(z)$ is the modular function of level 2 satisfying

$$
j(z)=2^{8}\left(\lambda^{2}-\lambda+1\right)^{3} / \lambda^{2}(\lambda-1)^{2},
$$

with values $(1, \infty, 0)$ at the cusps $(0,1, \infty)$. It is fixed by precisely the subgroup $\mathscr{Q}(2)$ of $\mathrm{GL}_{2}(\mathrm{~A})_{+}$.

Since curves having complex multiplication, isogenous over their field of definition, determine the same grössencharacter, to find the projective group of the Legendre and Jacobi families it suffices to find the projective group $P_{g}$ of the family g.

The modular function and the Jacobian elliptic functions can be expressed in terms of theta-functions as follows, writing $\vartheta_{r s}=\vartheta_{r s}(z)$ for $\theta(0, z, r, s)$.

$$
\begin{aligned}
\mathbf{k}^{2}(z) & =\frac{\vartheta_{(1 / 2) 0}(z)^{4}}{\vartheta_{00}(z)^{4}}, \\
s n\left(u, \mathbf{k}^{2}(z)\right) & =\frac{\vartheta_{00}}{\vartheta_{(1 / 2) 0}} \frac{\theta\left(u / \vartheta_{00}^{2}, z ; \frac{1}{2}, \frac{1}{2}\right)}{\theta\left(u / \vartheta_{00}^{2}, z ; 0, \frac{1}{2}\right)} \\
c n\left(u, \mathbf{k}^{2}(z)\right) & =\frac{\vartheta_{0(1 / 2)}}{\vartheta_{(1 / 2) 0}} \frac{\theta\left(u / \vartheta_{00}^{2}, z ; \frac{1}{2}, 0\right)}{\theta\left(u / \vartheta_{00}^{2}, z ; 0, \frac{1}{2}\right)}, \\
d n\left(u, \mathbf{k}^{2}(z)\right) & =\frac{\vartheta_{0(1 / 2)}}{\vartheta_{00}} \frac{\theta\left(u / \vartheta_{00}^{2}, z ; 0,0\right)}{\theta\left(u / \vartheta_{00}^{2}, z ; 0, \frac{1}{2}\right)}
\end{aligned}
$$

For our purposes the factor $\vartheta_{00}^{2}$ dividing the $u$-variable is irrelevant; a convenient equivalent form of the map parametrizing the family $\mathcal{G}$, in projective coordinates, is

$$
\begin{gathered}
J(u, z): \mathbf{C} \times \mathfrak{Q} \rightarrow \mathbf{P}^{3}, \\
J(u, z)=\left(\theta(u, z, 0,0), \frac{\vartheta_{00}}{\vartheta_{(1 / 2) 0}} \theta\left(u, z, \frac{1}{2} ; 0\right),\right. \\
\left.\frac{\vartheta_{00}}{\boldsymbol{\vartheta}_{0(1 / 2)}} \boldsymbol{\theta}\left(u, z ; 0, \frac{1}{2}\right), \frac{\vartheta_{00}}{\vartheta_{(1 / 2) 0}} \frac{\vartheta_{00}}{\vartheta_{0(1 / 2)}} \boldsymbol{\theta}\left(u, z ; \frac{1}{2}, \frac{1}{2}\right)\right) .
\end{gathered}
$$

Since the projective group of the family $q$ must fix $\mathbf{k}^{2}(z)$, we have that $\mathrm{GL}_{2}(\mathbf{Q})_{+}$ $\cap P_{\mathcal{g}} \subset \mathrm{GL}_{2}(\mathbf{Q})_{+} \cap \mathcal{U}(2)=\Gamma(2)$. Now we require a transformation formula, which 
is a consequence of (4a), (5) and (6); for future use we state it for arbitrary-dimensional theta-functions: for $r, s \in \frac{1}{2} \mathbf{Z}^{n}$ and $\gamma=\left(\begin{array}{ll}a & b \\ c & d\end{array}\right) \in \Gamma(2)$,

$$
\begin{aligned}
& \theta\left({ }^{t}(c z+d)^{-1} u, \gamma(z) ; r, s\right) \\
&= \lambda_{\gamma} e\left({ }^{t} r\left(\frac{a^{t} b}{2}\right) r-{ }^{t} r(a-1) s-{ }^{t} s\left(\frac{c^{t} d}{2}\right) s\right) \operatorname{det}(c z+d)^{1 / 2} \\
& \cdot e\left(\frac{1}{2}{ }^{t} u(c z+d)^{-1} c u\right) \cdot \theta(u, z ; r, s) .
\end{aligned}
$$

Applying this in the case $n=1$, we see that $\Gamma(4)$ and the elements $\left(\begin{array}{ll}1 & 2 \\ 0 & 1\end{array}\right),\left(\begin{array}{ll}1 & 0 \\ 2 & 1\end{array}\right)$ of $\Gamma(2)$ belong to $P_{g}$.

$\theta(u, z ; 0,0), \theta\left(u, z ; \frac{1}{2}, 0\right)$ and $\theta\left(u, z ; 0, \frac{1}{2}\right)$ are even functions of $u$ and have rational Fourier coefficients, while $\theta\left(u, z ; \frac{1}{2}, \frac{1}{2}\right)$ is odd and is $i$ times a function with rational Fourier coefficients. From formula (12) one sees that -1 fixes the even thetas and takes $\theta\left(u, z ; \frac{1}{2}, \frac{1}{2}\right)$ to its negative. Hence, with a slight abuse of notation,

$$
J(u, z)^{-1}=J(-u, z)
$$

and for $t \in \mathbf{Z}_{f}^{\times}$,

$$
J(u, z)^{\iota(t)}=J\left(\varepsilon_{t} u, z\right)
$$

where $\pm 1=\varepsilon_{t}=i^{[t, \mathbf{Q}]} / i \equiv t \bmod 4$. Combining these, we have that for $t \in \mathbf{Z}_{f}^{\times}$, $\iota(t) \in P_{g}$ if $t \equiv 1 \bmod 4$, and $-\iota(t) \in P_{g}$ if $t \equiv 3 \bmod 4$. Thus $\nu(P)=\mathbf{R}_{+} \times \mathbf{Z}_{f}^{\times}$. On the other hand $P$ contains an open subgroup of $\mathrm{GL}_{2}(\mathbf{A})_{+}$. Hence

THEOREM 3. The projective group of the Legendre and Jacobi families is the group

$$
P_{g}=\left\{u=\left(\begin{array}{ll}
a & b \\
c & d
\end{array}\right) \in \mathcal{Q} \mid u_{2} \equiv 1 \bmod 2 M_{2}\left(\mathbf{Z}_{2}\right), d_{2} \equiv 1 \bmod 4 Z_{2}\right\} .
$$

Furthermore the field $\mathfrak{I}_{P_{g}}$ is just $\mathbf{Q}(\lambda)=\mathbf{Q}\left(\mathbf{k}^{2}\right)$.

Proof. Indeed, $P_{g}$ has the same intersection with $G_{\mathbf{Q}^{+}}$as this group, and shares a common set of representatives of $\nu^{-1}\left(\mathbf{R}_{+}^{\times} \times \mathbf{Z}_{f}^{\times}\right)$, so by Proposition 4 the two groups are equal. Since now $\mathscr{U}(2)= \pm 1 \cdot P_{g}$, and all modular functions are fixed by $\pm \mathbf{1}, \mathfrak{f}_{P_{g}}=\mathbf{Q}(\lambda)$. The index $\left[\mathcal{Q}: P_{g}\right]=12$, the smallest possible value for a family defined at all points of $\mathfrak{Q}$.

(2) The Hesse curves, in homogeneous coordinates, are defined by the equation

$$
\mathcal{H}: x^{3}+y^{3}+w^{3}=3 \mu x y w .
$$

It is classical (see Bobek [2, p. 251]) that these curves can be parametrized by products of translates of any theta-function with a single zero in each period lattice; to obtain the largest possible projective group we will choose the particular basic theta

$$
\phi(u, z)=\prod_{m, n=0}^{1} \theta\left(u, z ; \frac{m}{2}, \frac{n}{2}\right) .
$$


As a function of $u, \phi(u, z)$ is a theta-function for the lattice $\frac{1}{2}[z, 1]$ with a simple zero at each lattice point. For $k=-1,0,1$ put

$$
\phi_{k}(u, z)=i \cdot e\left(\frac{16}{9} z\right) \cdot \phi\left(u-\frac{2}{3} z+\frac{2}{3} k, z\right) \cdot \phi\left(u+\frac{2}{3} k, z\right) \cdot \phi\left(u+\frac{2}{3} z+\frac{2}{3} k, z\right) .
$$

Then from (3), (5) and (6) one finds the functional equations

$$
\begin{gathered}
\phi_{k}\left(u+\frac{1}{2}, z\right)=-\phi_{k}(u, z), \quad \phi_{k}\left(u+\frac{1}{2} z, z\right)=-e\left(-\frac{3}{2} z-6 u\right) \cdot \phi_{k}(u, z), \\
\phi_{k}(-u, z)=-\phi_{-k}(u, z), \\
\phi_{k}\left(u+\frac{2}{3} z, z\right)=\zeta_{3}^{-k} \cdot e\left(-\frac{8}{3} z-8 u\right) \cdot \phi_{k}(u, z) .
\end{gathered}
$$

From these, in turn, it can be shown that

$$
\chi(u, z)=\frac{\phi_{-1}^{3}(u, z)+\phi_{0}^{3}(u, z)+\phi_{1}^{3}(u, z)}{\phi_{-1}(u, z) \cdot \phi_{0}(u, z) \cdot \phi_{1}(u, z)}
$$

is doubly periodic in $u$ for the lattice $\left[\frac{1}{6} z, \frac{1}{2}\right]$ and has no poles; hence it is independent of $u$. The projective embedding

$$
\begin{gathered}
H(u, z): \mathbf{C} \times \mathfrak{G} \rightarrow \mathbf{P}^{2}, \\
H(u, z)=\left(\phi_{-1}(u, z), \phi_{0}(u, z), \phi_{1}(u, z)\right)
\end{gathered}
$$

is a holomorphic isomorphism of $\mathbf{C} / \frac{1}{2}[z, 1]$ onto the curve $\mathcal{H}(z)$, where $\mu=\mu(z)$ $=\frac{1}{3} \chi(u, z)$.

We will now determine the projective group $P_{\mathcal{Y C}}$ of $H(u, z)$. From (5) and (6) and the definition of classical theta-functions,

$$
\phi_{k}(u, z)=i \prod_{l=-1}^{1} \prod_{m, n=0}^{1} \theta\left(u, z ; \frac{m}{2}+\frac{2}{3} l, \frac{n}{2}+\frac{2}{3} k\right)
$$

$$
=\prod_{l=-1}^{1} \prod_{m, n=0}^{1} \sum_{x \in \mathbf{Z}}(-1)^{n x} \zeta_{3}^{-k x} e\left(\frac{1}{2}\left(x+\frac{m}{2}+\frac{2}{3} l\right)^{2} z+\left(x+\frac{m}{2}+\frac{2}{3} l\right) u\right) .
$$

A calculation using (4), (5) and (6) now shows that $\Gamma(6) \subset P_{\mathcal{Y}}$. Actually $\Gamma(3)$ is contained in $P_{\mathfrak{I C}}$. To see this, we need the following supplement to formula (4b).

Recall formula (4b):

$\Theta\left({ }^{t}(c z+d)^{-1} u, \gamma(z) ; r, s\right)=\zeta \operatorname{det}(c z+d)^{1 / 2} e\left(\frac{1}{2}^{t} u(c z+d)^{-1} c u\right) \Theta\left(u, z, r^{\prime \prime}, s^{\prime \prime}\right)$.

LEMMA 1. The quantity $\zeta \cdot \operatorname{det}(c z+d)^{1 / 2}$ in (18) can be made explicit in the following cases:

$$
\begin{aligned}
& \text { if } \gamma=\left(\begin{array}{ll}
1 & 1 \\
0 & 1
\end{array}\right), \quad \zeta \cdot \operatorname{det}(c z+d)^{1 / 2}=\lambda_{\gamma} \cdot e\left(-\frac{1}{2}\left(r^{2}+r\right)\right)(c z+d)^{1 / 2}, \\
& \text { if } \gamma=\left(\begin{array}{ll}
1 & 0 \\
1 & 1
\end{array}\right), \quad \zeta \cdot \operatorname{det}(c z+d)^{1 / 2}=\lambda_{\gamma} \cdot e\left(-\frac{1}{2} s^{2}\right)(c z+d)^{1 / 2},
\end{aligned}
$$

where $\lambda_{\gamma}$ is an 8th root of unity depending only on $\gamma$. 
To obtain (19), expand $\theta(u, z+1 ; r, s)$ using the definition of classical thetafunction; to obtain (20), use (4a), (19), and the decomposition

$$
\left(\begin{array}{ll}
1 & 0 \\
1 & 1
\end{array}\right)=\left(\begin{array}{cc}
0 & -1 \\
1 & 0
\end{array}\right)\left(\begin{array}{cc}
1 & 1 \\
0 & 1
\end{array}\right)^{-1}\left(\begin{array}{cc}
0 & -1 \\
1 & 0
\end{array}\right)^{-1}
$$

Now a lengthy calculation using (16), (5), (6), (8),(19) and (20) shows that $\left(\begin{array}{ll}1 & 3 \\ 0 & 1\end{array}\right)$ and $\left(\begin{array}{ll}1 & 0 \\ 3 & 1\end{array}\right)$ belong to $P_{\mathcal{Y C}}$; these generate $\Gamma(3)$ over $\Gamma(6)$.

From (17) it is evident that for $t \in \mathbf{Z}_{f}^{x}$,

$$
\phi_{k}(u, z)^{\iota(t)}=\phi_{\varepsilon_{t}}(u, z) \quad \text { where } \pm 1=\varepsilon_{t} \equiv t \bmod 3 .
$$

From this it follows that $\mu(z)$ is a modular function with rational Fourier coefficients belonging to $\Gamma(3)$. Igusa $[4$, p. 456] gives the formula

$$
j(z)=3^{3} \frac{\mu^{3}\left(\mu^{3}+2\right)^{3}}{\left(\mu^{3}-1\right)^{3}}
$$

and a careful analysis of the $\phi_{k}(u, z)$ shows that $\mu(z)$ takes values $\left(\zeta_{3}^{-1}, \infty, \zeta_{3}, 1\right)$ at cusps $(-1,0,1, \infty)$. We see that $[\mathbf{Q}(\mu): \mathbf{Q}(j)]=12=[\mathcal{Q}: \mathcal{Q}(3)]$, so $\mathscr{U}(3)$ is the group fixing $\mathbf{Q}(\mu)$.

Combining (14) and (21), we have that for $t \in \mathbf{Z}_{f}^{\times}, \iota(t) \in P_{\mathscr{Y}}$ if $t \equiv 1 \bmod 3$, and $-\iota(t) \in P_{\mathcal{Y}}$ if $t \equiv 2 \bmod 3$.

THEOREM 4. The projective group of the family of Hesse curves is

$$
P_{\mathscr{K}}=\left\{u \in \mathcal{Q} \mid u_{3} \equiv\left(\begin{array}{cc} 
\pm 1 & 0 \\
0 & 1
\end{array}\right) \bmod 3 M_{2}\left(\mathbf{Z}_{3}\right)\right\}
$$

and $\mathfrak{H}_{P_{\mathcal{X}}}=\mathbf{Q}(\mu)$.

Proof. Again by Proposition 4, we see that this group $P$ is contained in $P_{\mathscr{F}}$. However, $\mathscr{Q}(3)= \pm \mathbf{1} \cdot P$, and $P_{\mathcal{Y C}}$ fixes $\mathbf{Q}(\mu)$, which is fixed by precisely $\mathcal{Q}$ (3). Since

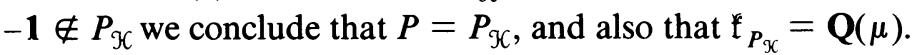

B. A two-dimensional example. We now turn to the case $n=2$ and consider abelian varieties parametrized by the $\theta(u, z ; r, s)$ with $r, s \in \frac{1}{2} \mathbf{Z}^{2}$. If $g$ is a set of representatives for $\frac{1}{2} \mathbf{Z}^{2} \times \mathbf{Z}^{2} / \mathbf{Z}^{2} \times \mathbf{Z}^{2}$, then it is known [10,p. 682] that for any $z \in \mathfrak{Q}_{2}$,

$$
u \mapsto(\theta(u, z ; r, s))_{(r, s) \in \mathcal{G}}
$$

gives a nonsingular projective embedding of $\mathbf{C}^{2} / 2(z \mathbf{1}) \mathbf{Z}^{4}$. Unfortunately this family has a relatively small projective group: one sees, using (12) above, that the largest subgroup of $\operatorname{Sp}(2, \mathbf{Z})$ contained in it is $\left\{\left(\begin{array}{ll}a & b \\ c & d\end{array}\right) \in \Gamma(4) \mid b \equiv c \equiv 0 \bmod 8 M_{2}(Z)\right\}$.

To obtain a larger projective group, we use an embedding modeled on the one obtained above from the Jacobian elliptic functions. Of the sixteen $\theta(u, z ; r, s)$ with $(r, s) \in \mathcal{G}$, ten are even functions of $u$ and six are odd. Suppose $z$ is a point where all ten even thetas are nonvanishing at $u=0$ (this is the generic case) and consider the embedding $\Phi(u, z)$ given by the functions $\phi(u, z ; r, s)$ in Table 1 below. (For compactness of notation, we have written

$\vartheta\left(\begin{array}{ll}r_{1} & s_{1} \\ r_{2} & s_{2}\end{array}\right)=\theta\left(0, z ;\left(\begin{array}{l}r_{1} \\ r_{2}\end{array}\right),\left(\begin{array}{l}s_{1} \\ s_{2}\end{array}\right)\right)$ and $\theta\left(u, z ; \begin{array}{ll}r_{1} & s_{1} \\ r_{2} & s_{2}\end{array}\right)=\theta\left(u, z ;\left(\begin{array}{l}r_{1} \\ r_{2}\end{array}\right),\left(\begin{array}{l}s_{1} \\ s_{2}\end{array}\right)\right)$. 
The first ten $\phi(u, z ; r, s)$ arise from the even theta-functions, and the last six from the odd ones.)

Since the even thetas have rational Fourier coefficients, while each odd theta is $i$ times a function with rational Fourier coefficients (as can be seen from their definition), $\iota\left(\mathbf{Z}_{f}^{\times}\right)$is contained in the projective group $P$ of $\Phi(u, z) . P$ also contains the following subgroup of $\operatorname{Sp}(2, Z)$, which has index 2 in $\Gamma(2)$ :

$$
\Gamma_{P}=\left\{\gamma=\left(\begin{array}{ll}
a & b \\
c & d
\end{array}\right) \in \Gamma(2) \mid a=\left(\begin{array}{ll}
a_{11} & a_{12} \\
a_{21} & a_{22}
\end{array}\right) \equiv 1 \bmod 2 \text {, with } a_{11} \equiv 1 \bmod 4\right\} .
$$

Indeed, if $\phi(u, z)$ is any one of the functions in Table 1 , and $\gamma \in \Gamma_{P}$, then

$$
\phi\left({ }^{t}(c z+d)^{-1} u, \gamma(z)\right)=\lambda_{\gamma} \operatorname{det}(c z+d)^{1 / 2} e\left(\frac{1}{2}^{t} u(c z+d)^{-1} c u\right) \phi(u, z) .
$$

This follows from equation (12): the essential point is that the modifying factors have been chosen so that every parameter $r=\left(\begin{array}{l}r_{1} \\ r_{2}\end{array}\right)$ or $s=\left(\begin{array}{l}s_{1} \\ s_{2}\end{array}\right)$ which occurs in a theta in the numerator also occurs in one in the denominator. Hence all terms of the type $e\left({ }^{t} r\left(a^{t} b / 2\right) r\right)$ and $e\left({ }^{t} s\left(c^{t} d / 2\right) s\right)$ from equation (12) cancel. If $\phi(u, z)$ is derived from one of the even thetas, (22) obviously holds for all $\gamma \in \Gamma(2)$. The modifying factors for the odd thetas have been chosen so that the terms $e\left(-{ }^{t} r(a-1) s\right)$ cancel as much as possible.

Since the abelian varieties parametrized by $\Phi(u, z)$ are principally polarized, it follows that $P \subset \mathcal{Q}$; and also by the transformation formula (4b), any $\gamma \in \operatorname{Sp}(2, Z)$ which belongs to $P$ must belong to $\Gamma(2)$. Hence $G_{\mathbf{Q}^{+}} \cap P=\Gamma_{P}$, and by Proposition 4,

THEOREM 5. The projective group of the family of Table 1 is

$$
\begin{aligned}
& P=\left\{u=\left(\begin{array}{ll}
a & b \\
c & d
\end{array}\right) \in \mathcal{Q} \mid u_{2} \equiv 1 \bmod 2 M_{4}\left(\mathbf{Z}_{2}\right)\right. \\
&\left.a_{2}=\left(\begin{array}{ll}
a_{11} & a_{12} \\
a_{21} & a_{22}
\end{array}\right) \text { with } a_{11} \equiv 1 \bmod 4 Z_{2}\right\} .
\end{aligned}
$$

The index $[\mathscr{Q}: P]=2^{5} 3^{2} 5$.

\section{TABle 1. Modified TheTA-FunCtions}

$$
\begin{aligned}
& \theta\left(u, z ; \begin{array}{ll}
0 & 0 \\
0 & 0
\end{array}\right) \\
& \frac{\vartheta\left(\begin{array}{cc}
0 & 0 \\
0 & 0
\end{array}\right)}{\vartheta\left(\begin{array}{cc}
\frac{1}{2} & 0 \\
0 & \frac{1}{2}
\end{array}\right)} \boldsymbol{\theta}\left(u, z ; \begin{array}{cc}
\frac{1}{2} & 0 \\
0 & \frac{1}{2}
\end{array}\right) \\
& \frac{\vartheta\left(\begin{array}{ll}
0 & 0 \\
0 & 0
\end{array}\right)}{\vartheta\left(\begin{array}{ll}
\frac{1}{2} & 0 \\
0 & 0
\end{array}\right)} \theta\left(u, z ; \begin{array}{cc}
\frac{1}{2} & 0 \\
0 & 0
\end{array}\right) \\
& \frac{\vartheta\left(\begin{array}{cc}
0 & 0 \\
0 & 0
\end{array}\right)}{\vartheta\left(\begin{array}{ll}
0 & \frac{1}{2} \\
\frac{1}{2} & 0
\end{array}\right)} \boldsymbol{\theta}\left(u, z ; \begin{array}{cc}
0 & \frac{1}{2} \\
\frac{1}{2} & 0
\end{array}\right) \\
& \frac{\vartheta\left(\begin{array}{ll}
0 & 0 \\
0 & 0
\end{array}\right)}{\vartheta\left(\begin{array}{ll}
0 & \frac{1}{2} \\
0 & 0
\end{array}\right)} \theta\left(u, z ; \begin{array}{cc}
0 & \frac{1}{2} \\
0 & 0
\end{array}\right) \\
& \frac{\vartheta\left(\begin{array}{ll}
0 & 0 \\
0 & 0
\end{array}\right)}{\vartheta\left(\begin{array}{cc}
\frac{1}{2} & 0 \\
\frac{1}{2} & 0
\end{array}\right)} \theta\left(u, z ; \begin{array}{cc}
\frac{1}{2} & 0 \\
\frac{1}{2} & 0
\end{array}\right)
\end{aligned}
$$




$$
\begin{aligned}
& \frac{\vartheta\left(\begin{array}{ll}
0 & 0 \\
0 & 0
\end{array}\right)}{\vartheta\left(\begin{array}{ll}
0 & 0 \\
\frac{1}{2} & 0
\end{array}\right)} \theta\left(u, z ; \frac{1}{2} \quad 0\right) \\
& \frac{\vartheta\left(\begin{array}{ll}
0 & 0 \\
0 & 0
\end{array}\right)}{\vartheta\left(\begin{array}{ll}
0 & \frac{1}{2} \\
0 & \frac{1}{2}
\end{array}\right)} \theta\left(u, z ; \begin{array}{cc}
0 & \frac{1}{2} \\
0 & \frac{1}{2}
\end{array}\right) \\
& \frac{\vartheta\left(\begin{array}{ll}
0 & 0 \\
0 & 0
\end{array}\right)}{\vartheta\left(\begin{array}{ll}
0 & 0 \\
0 & \frac{1}{2}
\end{array}\right)} \theta\left(u, z ; \begin{array}{cc}
0 & 0 \\
0 & \frac{1}{2}
\end{array}\right) \\
& \frac{\vartheta\left(\begin{array}{ll}
0 & 0 \\
0 & 0
\end{array}\right)}{\vartheta\left(\begin{array}{cc}
\frac{1}{2} & \frac{1}{2} \\
\frac{1}{2} & \frac{1}{2}
\end{array}\right)} \theta\left(u, z ; \begin{array}{cc}
\frac{1}{2} & \frac{1}{2} \\
\frac{1}{2} & \frac{1}{2}
\end{array}\right) \\
& i \frac{\vartheta\left(\begin{array}{ll}
0 & 0 \\
0 & 0
\end{array}\right)}{\vartheta\left(\begin{array}{ll}
\frac{1}{2} & 0 \\
0 & 0
\end{array}\right)} \frac{\vartheta\left(\begin{array}{ll}
0 & 0 \\
0 & 0
\end{array}\right)}{\vartheta\left(\begin{array}{ll}
0 & \frac{1}{2} \\
0 & 0
\end{array}\right)} \theta\left(u, z ; \begin{array}{cc}
\frac{1}{2} & \frac{1}{2} \\
0 & 0
\end{array}\right) \\
& i \frac{\vartheta\left(\begin{array}{ll}
0 & 0 \\
0 & 0
\end{array}\right)}{\vartheta\left(\begin{array}{cc}
\frac{1}{2} & 0 \\
\frac{1}{2} & 0
\end{array}\right)} \frac{\vartheta\left(\begin{array}{cc}
\frac{1}{2} & \frac{1}{2} \\
\frac{1}{2} & \frac{1}{2}
\end{array}\right)}{\vartheta\left(\begin{array}{ll}
0 & \frac{1}{2} \\
0 & \frac{1}{2}
\end{array}\right)} \frac{\vartheta\left(\begin{array}{cc}
0 & \frac{1}{2} \\
0 & 0
\end{array}\right)}{\vartheta\left(\begin{array}{ll}
0 & \frac{1}{2} \\
\frac{1}{2} & 0
\end{array}\right)} \frac{\vartheta\left(\begin{array}{cc}
\frac{1}{2} & 0 \\
0 & 0
\end{array}\right)}{\vartheta\left(\begin{array}{cc}
\frac{1}{2} & 0 \\
0 & \frac{1}{2}
\end{array}\right)} \theta\left(u, z ; \begin{array}{cc}
0 & 0 \\
\frac{1}{2} & \frac{1}{2}
\end{array}\right) \\
& i \frac{\vartheta\left(\begin{array}{ll}
0 & 0 \\
0 & 0
\end{array}\right)}{\vartheta\left(\begin{array}{ll}
\frac{1}{2} & 0 \\
0 & 0
\end{array}\right)} \frac{\vartheta\left(\begin{array}{cc}
\frac{1}{2} & 0 \\
0 & \frac{1}{2}
\end{array}\right)}{\vartheta\left(\begin{array}{ll}
0 & 0 \\
0 & \frac{1}{2}
\end{array}\right)} \frac{\vartheta\left(\begin{array}{ll}
0 & 0 \\
0 & 0
\end{array}\right)}{\vartheta\left(\begin{array}{cc}
0 & 0 \\
\frac{1}{2} & 0
\end{array}\right)} \frac{\vartheta\left(\begin{array}{cc}
\frac{1}{2} & 0 \\
\frac{1}{2} & 0
\end{array}\right)}{\vartheta\left(\begin{array}{cc}
\frac{1}{2} & \frac{1}{2} \\
\frac{1}{2} & \frac{1}{2}
\end{array}\right)} \theta\left(u, z ; \begin{array}{cc}
0 & \frac{1}{2} \\
\frac{1}{2} & \frac{1}{2}
\end{array}\right) \\
& i \frac{\vartheta\left(\begin{array}{ll}
0 & 0 \\
0 & 0
\end{array}\right)}{\vartheta\left(\begin{array}{cc}
0 & 0 \\
\frac{1}{2} & 0
\end{array}\right)} \frac{\vartheta\left(\begin{array}{cc}
0 & \frac{1}{2} \\
\frac{1}{2} & 0
\end{array}\right)}{\vartheta\left(\begin{array}{ll}
0 & \frac{1}{2} \\
0 & 0
\end{array}\right)} \frac{\vartheta\left(\begin{array}{ll}
0 & 0 \\
0 & 0
\end{array}\right)}{\vartheta\left(\begin{array}{ll}
0 & 0 \\
0 & \frac{1}{2}
\end{array}\right)} \frac{\vartheta\left(\begin{array}{cc}
0 & \frac{1}{2} \\
0 & \frac{1}{2}
\end{array}\right)}{\vartheta\left(\begin{array}{cc}
\frac{1}{2} & \frac{1}{2} \\
\frac{1}{2} & \frac{1}{2}
\end{array}\right)} \theta\left(u, z ; \begin{array}{cc}
\frac{1}{2} & 0 \\
\frac{1}{2} & \frac{1}{2}
\end{array}\right) \\
& i \frac{\vartheta\left(\begin{array}{cc}
0 & 0 \\
\frac{1}{2} & 0
\end{array}\right)}{\vartheta\left(\begin{array}{cc}
0 & \frac{1}{2} \\
\frac{1}{2} & 0
\end{array}\right)} \frac{\vartheta\left(\begin{array}{ll}
0 & 0 \\
0 & 0
\end{array}\right)}{\vartheta\left(\begin{array}{cc}
\frac{1}{2} & 0 \\
\frac{1}{2} & 0
\end{array}\right)} \theta\left(u, z ; \begin{array}{cc}
\frac{1}{2} & \frac{1}{2} \\
\frac{1}{2} & 0
\end{array}\right) \\
& i \frac{\vartheta\left(\begin{array}{cc}
0 & 0 \\
0 & \frac{1}{2}
\end{array}\right)}{\vartheta\left(\begin{array}{cc}
\frac{1}{2} & 0 \\
0 & \frac{1}{2}
\end{array}\right)} \frac{\vartheta\left(\begin{array}{ll}
0 & 0 \\
0 & 0
\end{array}\right)}{\vartheta\left(\begin{array}{cc}
0 & \frac{1}{2} \\
0 & \frac{1}{2}
\end{array}\right)} \theta\left(u, z ; \begin{array}{cc}
\frac{1}{2} & \frac{1}{2} \\
0 & \frac{1}{2}
\end{array}\right)
\end{aligned}
$$

We will now determine the fields of definition and grössencharacters given by Theorem 1 for two specific varieties in this family. In both cases the ten even $\theta(u, z ; r, s)$ are numerically found to be nonzero. 
Recall [16, p. 50] that if we are given a CM field $K$ of degree $2 n$, embeddings $\sigma_{1}, \ldots, \sigma_{n}$ of $K$ into $\mathrm{C}$ no two of which are complex conjugate, and a lattice $L \subset K$, then putting

$$
u(k)=\left(\begin{array}{c}
k^{\sigma_{1}} \\
\vdots \\
k^{\sigma_{n}}
\end{array}\right) \quad \text { for } k \in K
$$

the torus $\mathbf{C}^{n} / u(L)$ is an abelian variety $A$ having CM type $\left(K,\left\{\sigma_{1}, \ldots, \sigma_{n}\right\}\right)$. Every polarization $P$ of $A$ is induced by an element $\beta$ of $K$ whose image under each of $\sigma_{1}, \ldots, \sigma_{n}$ lies on the positive imaginary axis: namely, its associated Riemann form is

$$
E(u(a), u(b))=\operatorname{Tr}_{K / \mathbf{Q}}\left(\beta a b^{\rho}\right) \quad \text { for } a, b \in K,
$$

where $\rho$ is the complex conjugation.

If such a $\beta$ is given, and $x_{1}, \ldots, x_{2 n}$ is an ordered basis of $L$ such that the $2 n \times 2 n$ matrix $\left(\operatorname{Tr}_{K / Q}\left(\beta x_{i} x_{j}^{\rho}\right)\right)$ is $J$, then $P$ is principal. Moreover, by the recipe at the beginning of this section, if $\Omega=\left(\omega_{1} \omega_{2}\right)$ is the matrix whose columns are $u\left(x_{1}\right), \ldots, u\left(x_{2 n}\right)$, the point in $\mathfrak{Q}_{n}$ corresponding to $(A, P)$ is $z_{0}=\omega_{2}^{-1} \omega_{1}$, and the embedding $\xi: K \rightarrow M_{2 n}(\mathbf{Q})$ at the fixed point $z_{0}$ is determined by

$$
\left(\begin{array}{ccc}
k^{\sigma_{1}} & & \\
& \ddots & \\
& & k^{\sigma_{n}}
\end{array}\right) \Omega=\Omega^{t} \xi(k) \quad \text { for } k \in K
$$

(1) Our family contains a twisted form of the Jacobian of $y^{2}=1-x^{5}$, which admits complex multiplication by the maximal order of $\mathbf{Q}\left(\zeta_{5}\right)$ and is canonically principally polarized. It is isomorphic to $C^{2} / u\left(\theta_{K}\right)$ and has $C M$ type $\left(K,\left\{\sigma_{1}, \sigma_{2}\right\}\right)$, where $\sigma_{j}: K=\mathbf{Q}\left(\zeta_{5}\right) \rightarrow \mathbf{C}$ takes $\zeta=\zeta_{5}$ to $e(j / 5)$. One checks that $\beta=\left(\zeta-\zeta^{-1}\right) / 5$ and the ordered basis $\zeta^{2}, \zeta^{4}, \zeta, \zeta+\zeta^{3}$ of $\theta_{K}$ satisfy the conditions above, giving

$$
z_{0}=\left(\begin{array}{cc}
\zeta^{2}-\zeta^{3} & \zeta+\zeta^{3}+\zeta^{4} \\
\zeta+\zeta^{3}+\zeta^{4} & -\zeta^{4}
\end{array}\right), \quad \xi(\zeta)=\left(\begin{array}{cccc}
0 & 0 & -1 & 1 \\
-1 & -1 & 0 & -1 \\
1 & 0 & 0 & 0 \\
1 & 1 & 0 & 0
\end{array}\right) .
$$

$\left(K ;\left\{\sigma_{1}, \sigma_{2}\right\}\right)$ is its own reflex, that is, $K^{\prime}=K . K^{\prime}=\mathbf{Q}\left(\xi_{5}\right)$ and its totally real subfield $F^{\prime}=\mathbf{Q}(\sqrt{5})$ have class number 1 . All units of $K^{\prime}$ are of the form $\pm \zeta^{c} \omega^{d}$ where $\omega=\frac{1}{2}(1+\sqrt{5})$ is the fundamental unit of $F^{\prime} ; \omega$ has norm -1 . The prime (2) is inert in $K^{\prime} / \mathbf{Q}$; we write $\mathfrak{p}_{2}$ for $2 \vartheta_{K}$.

We want to find $(\xi \circ \eta)^{-1}(P)$ where $\eta: K_{\mathbf{A}}^{\prime \times} \rightarrow K_{\mathbf{A}}^{\times}$is given by $\eta(k)=k^{\sigma_{1}} k^{\sigma_{2}}$. By direct calculation the part of $(\xi \circ \eta)^{-1}(P)$ contained in the unit subgroup of $K_{\mathbf{A}}^{\prime \times}$ is

$$
S_{0}=\mathbf{C}^{\times} \times \mathbf{C}^{\times} \times\left[\left\{-1, \omega^{2}, 1+2 \zeta^{2}, 1+2 \zeta+2 \zeta^{3}\right\} \cdot\left(1+p_{2}^{2}\right)\right] \times \prod_{\mathfrak{p} \nmid 2} \mathcal{u}_{p} .
$$

Here the quantity in brackets means the subgroup of $K_{\mathfrak{p}_{2}}^{\prime \times}$ generated over $1+\mathfrak{p}_{2}^{2}$ by the given elements. $S_{0}$ has index 10 in $\mathbf{C}^{\times} \times \mathbf{C}^{\times} \times \Pi_{p} \mathscr{q}_{p}$ and coset representatives are given by the $\zeta^{c} \omega^{d}$; hence $K_{\mathrm{A}}^{\prime \times} \cdot S_{0}$. Decomposing an arbitrary $x \in(\xi \circ \eta)^{-1}(P)$ as $x=k^{\prime} \cdot s$ with $k^{\prime} \in K^{\prime}, s \in S_{0}$, we find $(\xi \circ \eta)\left(k^{\prime}\right) \in P \cap(\xi \circ \eta)\left(K^{\prime \times}\right)=\{1\}$. As $\xi$ is injective, $\eta\left(k^{\prime}\right)=1$. Let $\left(k^{\prime}\right)$ be the ideal of $K^{\prime}$ generated by $k^{\prime}$ : so $\left(k^{\prime}\right)^{\sigma_{1}} \cdot\left(k^{\prime}\right)^{\sigma_{2}}=$ (1). Examination of the galois action on primes in $K^{\prime} / \mathbf{Q}$ shows that $\left(k^{\prime}\right)$ is an ideal 
of $F^{\prime}: k^{\prime}=\zeta^{c} f$ with some $c \in \mathbf{Z}, f \in F^{\prime}$. But $1=\eta\left(k^{\prime}\right)=\zeta^{3 c} N_{F^{\prime} / \mathbf{Q}}(f)$ forces $k^{\prime}=f$;

$$
(\xi \circ \eta)^{-1}(P)=N_{F^{\prime} / Q}^{-1}(1) \cdot S_{0} \text {. }
$$

In the course of the analysis we have shown that the variety is defined over $K^{\prime}=\mathbf{Q}\left(\zeta_{5}\right)$ itself, the classfield to $K_{\mathbf{A}}^{\prime \times}$. By the theorem, the grössencharacters $\psi_{1}, \psi_{2}: K_{\mathbf{A}}^{\prime \times} \rightarrow \mathbf{C}^{\times}$belonging to it are

$$
\psi_{1}(x)=\frac{\eta\left(k^{\prime}\right)^{\sigma_{1}}}{\eta(x)_{\infty_{1}}}, \quad \psi_{2}(x)=\frac{\eta\left(k^{\prime}\right)^{\sigma_{2}}}{\eta(x)_{\infty_{2}}}
$$

for $x \in K_{\mathrm{A}}^{\prime \times}$, where $x=k^{\prime} \cdot s$ with some $k^{\prime} \in K^{\prime \times}$ and $s \in(\xi \circ \eta)^{-1}(P)$, and $\infty_{1}$ and $\infty_{2}$ are the archimedean primes of $K$ corresponding to the embeddings $\sigma_{1}$ and $\sigma_{2}$ respectively.

(2) For a second example, take

$$
K=\mathbf{Q}(\sqrt{3+\sqrt{2}} i)
$$

and consider the torus $\mathrm{C}^{2} / u\left(\vartheta_{K}\right)$ with $\mathrm{CM}$ type $\left(K,\left\{\sigma_{1}, \sigma_{2}\right\}\right)$, where $\sigma_{1}$ and $\sigma_{2}$ are chosen so that

$$
\operatorname{Im}\left((\sqrt{3+\sqrt{2}} i)^{\sigma_{j}}\right)>0 .
$$

This is a "dihedral" example: $K$ is not normal over $\mathbf{Q}$; the galois group of its galois closure is the dihedral group with 8 elements. The torus is principally polarized by

$$
\beta=\frac{1}{28}\left(2+\frac{\sqrt{2}}{2}\right) \sqrt{3+\sqrt{2}} i,
$$

and through the ordered basis

$$
(2-\sqrt{2}) \sqrt{3+\sqrt{2}} i,(-1+\sqrt{2}) \sqrt{3+\sqrt{2}} i, 1, \sqrt{2}
$$

of $\vartheta_{K}$, we obtain the point and embedding

$$
z_{0}=\left(\begin{array}{cc}
2+\sqrt{7} & -\frac{1}{2}-\frac{1}{2} \sqrt{7} \\
-\frac{1}{2}-\frac{1}{2} \sqrt{7} & 1+\frac{1}{2} \sqrt{7}
\end{array}\right) \sqrt{3-\sqrt{7}} i, \quad \xi(\sqrt{3+\sqrt{2}} i)=\left(\begin{array}{cccc}
0 & 0 & -4 & 1 \\
0 & 0 & 1 & -2 \\
1 & 1 & 0 & 0 \\
1 & 2 & 0 & 0
\end{array}\right) .
$$

The reflex to $\left(K,\left\{\sigma_{1}, \sigma_{2}\right\}\right)$ is $\left(K^{\prime},\left\{\nu_{1}, \nu_{2}\right\}\right)$ where $K^{\prime}=\mathbf{Q}(\sqrt{3-\sqrt{7}} i)$ and $\nu_{1}$ and $\nu_{2}$ take $\sqrt{3-\sqrt{7}} i$ to the positive imaginary axis. $K^{\prime}$ has class number 2 ; its totally real subfield $F^{\prime}=\mathbf{Q}(\sqrt{7})$ has class number 1 . (2) is totally ramified in $K^{\prime}$; the prime $\mathfrak{p}_{2}$ above it is principal: $\sqrt{3-\sqrt{7}} i$ is a generator.

By direct computation, the subgroup of the unit group of $K_{A}^{\prime \times}$ contained in $(\xi \circ \eta)^{-1}(P)$ may be found to be

$$
S_{0}=\mathbf{C}^{\times} \times \mathbf{C}^{\times} \times\left(1+\mathfrak{p}_{2}^{2}\right) \times \prod_{\mathfrak{p}+2} \mathfrak{u}_{\mathfrak{p}},
$$

and an argument similar to the previous one, based on [13, Proposition A.7, p. 84] gives

$$
(\xi \circ \eta)^{-1}(P)=N_{F^{\prime} / \mathbf{Q}}^{-1}(1) \cdot S_{0} .
$$


The variety is defined over the classfield to $K^{\prime \times} \cdot(\xi \circ \eta)^{-1}(P)$, which can be shown to be

$$
\mathbf{k}=K^{\prime}(\sqrt{2-\sqrt{7}+2 \sqrt{3-\sqrt{7}}})
$$

it is a cyclic extension of $K^{\prime}$ of degree 4 . The grössencharacters $\psi_{1}, \psi_{2}: \mathbf{k}_{\mathbf{A}}^{\times} \rightarrow \mathbf{C}^{\times}$are

$$
\psi_{1}(x)=\frac{\eta\left(k^{\prime}\right)^{\sigma_{1}}}{\eta\left(N_{\mathbf{k} / K^{\prime}}(x)\right)_{\infty_{1}}}, \quad \psi_{2}(x)=\frac{\eta\left(k^{\prime}\right)}{\eta\left(N_{\mathbf{k} / K^{\prime}}(x)\right)_{\infty_{2}}}
$$

for $x \in \mathbf{k}_{\mathbf{A}}^{\times}$, where $N_{\mathbf{k} / K^{\prime}}(x)=k^{\prime} \cdot s$, with $k^{\prime} \in K^{\prime \times}, s \in(\xi \circ \eta)^{-1}(P)$ as above.

C. The STANDARD EMBEDdings. For theoretical purposes, it may be useful to know (a subgroup of) the projective group for a family of abelian varieties with specified dimension and polarization type. Using different terminology, Shimura [11, Theorem 1.1, p. 368] has determined such a subgroup for the families

$$
\Theta_{\varepsilon, w}(u, z)=(\theta(w u, w z ; j, 0))_{j \in \mathcal{g}}
$$

mentioned after Proposition 2 above; here $\varepsilon$ is the specified matrix of elementary divisors, $w \geqslant 3$ is an integer, and $g$ is a set of representatives for $\delta^{-1} \mathbf{Z}^{n} / \mathbf{Z}^{n}$, where $\delta=w \cdot \varepsilon$. We reformulate his result in the context of Theorem 3 .

First, writing $x \in G_{\mathbf{A}^{+}}$as $x=\left(\begin{array}{ll}a & b \\ c & d\end{array}\right)$, let

$$
\begin{gathered}
S_{\theta}=\left\{x \in G_{\mathbf{A}^{+}} \mid x_{p} \in \mathrm{GL}_{2 n}\left(\mathbf{Z}_{p}\right) \forall p ;\left\{a_{2}{ }^{t} b_{2}\right\} \equiv\left\{c_{2}{ }^{t} d_{2}\right\} \equiv 0 \bmod 2 M_{2}\left(\mathbf{Z}_{2}\right)\right\}, \\
S_{\delta}=\left\{x \in S_{\theta} \cap\left(\begin{array}{cc}
\delta & 0 \\
0 & \delta^{-1}
\end{array}\right) S_{\theta}\left(\begin{array}{cc}
\delta^{-1} & 0 \\
0 & \delta
\end{array}\right) \mid a_{p}-1 \in M_{n}\left(\mathbf{Z}_{p}\right) \text { for all } p\right\} .
\end{gathered}
$$

THEOREM 6. The projective group of $\Theta_{\varepsilon, w}(u, z)$ contains the subgroup of $G_{\mathbf{A}^{+}}$

$$
T_{\varepsilon, w}=\left(\begin{array}{cc}
w 1 & 0 \\
0 & 1
\end{array}\right)^{-1} S_{\delta}\left(\begin{array}{cc}
w 1 & 0 \\
0 & 1
\end{array}\right) \text {. }
$$

Proof. By general facts about conjugation of subgroups, it suffices to show that $S_{\delta}$ is contained in the projective group $P_{\delta}$ of

$$
\Theta_{\varepsilon, w}^{\prime}(u, z)=(\theta(u, z ; j, 0))_{j \in g} .
$$

Now $S_{\delta}$ is generated over any open subgroup by $\iota\left(\mathbf{Z}_{f}^{\times}\right)$and $\Gamma_{\delta}=S_{\delta} \cap \operatorname{Sp}(n, \mathbf{Z})$, and $\iota\left(\mathbf{Z}_{f}^{\times}\right) \subset P_{\delta}$ since the $\theta(u, z ; j, 0)$ have rational Fourier coefficients, so it suffices to show $\Gamma_{\delta} \subset P_{\delta}$.

Note that $\Gamma_{\theta}$ is stable under transposes. Writing $\gamma \in \Gamma_{\delta}$ as

$$
\gamma=\left(\begin{array}{ll}
a & b \\
c & d
\end{array}\right)=\left(\begin{array}{cc}
\delta a_{1} \delta^{-1} & \delta b_{1} \delta \\
\delta^{-1} c_{1} \delta^{-1} & \delta^{-1} d_{1} \delta
\end{array}\right) \quad \text { with }\left(\begin{array}{ll}
a_{1} & b_{1} \\
c_{1} & d_{1}
\end{array}\right) \in \Gamma_{\theta},
$$

by equations (4) and (6) we have

$$
\begin{aligned}
& \theta\left({ }^{t}(c z+d)^{-1} u, \gamma(z) ; j, 0\right) \\
&= \lambda_{\gamma} e\left(-\frac{1}{2}^{t} j a^{t} b j\right) \operatorname{det}(c z+d)^{1 / 2} e\left(\frac{1}{2}^{t} u(c z+d)^{-1} c u\right) \theta\left(u, z ;{ }^{t} a j,{ }^{t} b j\right) \\
&= \lambda_{\gamma} e\left(-\frac{1}{2}^{t}(\delta j) a_{1}^{t} b_{1}(\delta j)\right) e\left({ }^{t} j(a-1) \delta^{t} b_{1} \delta j\right) \operatorname{det}(c z+d)^{1 / 2} \\
& \cdot e\left(\frac{1}{2}^{t} u(c z+d)^{-1} c u\right) \theta(u, z ; j, 0) .
\end{aligned}
$$


The first exponential term is trivial since $\delta j \in \mathbf{Z}^{n}$ and $a_{1}{ }^{t} b_{1}$ is a symmetric matrix with even diagonal; the second is trivial since $a-1$ and $\delta^{t} b_{1} \delta$ belong to $\delta M_{n}(\mathbf{Z})$. This means that $\gamma$ lifts to an element of $\mathcal{G}_{\mathbf{A}^{+}}$fixing all the $\theta(u, z ; j, 0)$ for $j \in \mathcal{G}$.

We remark that $T_{\varepsilon, w}$ need not be the full projective group even in the simplest cases: for example, if $n=2, \varepsilon=1$, and $w=4$, then it is easily seen that

$$
\gamma=\left(\begin{array}{cccc}
1 & 0 & 16 & 8 \\
0 & 1 & 8 & 16 \\
0 & 0 & 1 & 0 \\
0 & 0 & 0 & 1
\end{array}\right) \in P_{\delta} \backslash S_{\delta}
$$

The conclusions of Theorem 1 remain valid with $T_{\varepsilon, w}$ replacing the full projective group, but the fields of definition produced may be larger than necessary.

\section{REFERENCES}

1. B. J. Birch and W. Kuyk, Eds., Numerical tables on elliptic curves, Table 3, Lecture Notes in Math., vol. 476, Springer-Verlag, Berlin and New York, 1975, pp. 116-122.

2. K. J. Bobek, Einleitung in die Theorie der Elliptischen Funktionen, Teubner, Leipzig, 1884.

3. M. Deuring, Die Zetafunktion einer algebraischen Kurve vom Geschlechte Eins. I, II, III, IV, Nachr. Akad. Wiss. Göttingen (1953), 85-94; (1955), 13-42; (1956), 37-76; (1957), 55-80.

4. J-C. Igusa, Fibre systems of Jacobian varieties. III, Amer. J. Math. 81 (1959), 453-476.

5. S. Lang, Elliptic functions, Addison-Wesley, Reading, Mass., 1973.

6. A. Ogg, Elliptic curves and wild ramifications, Amer. J. Math. 89 (1967), 1-21.

7. R. Rumely, A formula for the grössencharacter of a parametrized elliptic curve, J. Number Theory. (to appear).

8. G. Shimura, Introduction to the arithmetic theory of automorphic functions, Iwanami Shoten/Princeton Univ. Press, Princeton, N.J., 1971.

9. On canonical models of arithmetic quotients of bounded symmetric domains. I, II, Ann. of Math. (2) 91 (1970), 144-222; 92 (1970), 528-549.

10. __ Theta functions with complex multiplication, Duke Math. J. 43 (1976), 673-696.

11. On the derivatives of theta functions and modular forms, Duke Math. J. 44 (1977), 365-387.

12. __ On certain reciprocity laws for theta functions and modular forms, Acta Math. 141 (1978), $35-71$.

13. 65-86. , On abelian varieties with complex multiplication, Proc. London Math. Soc. (3) 34 (1977),

14. On elliptic curves with complex multiplication as factors of the Jacobians of modular function fields, Nagoya Math. J. 43 (1971), 199-208.

15. On the zeta function of an abelian variety with complex multiplication, Ann. of Math. (2) 94 (1971), 504-533.

16. G. Shimura and Y. Taniyama, Complex multiplication of abelian varieties and its application to number theory, Math. Soc. Japan, 1961.

17. J-P. Serre and J. Tate, Good reduction of abelian varieties, Ann. of Math. (2) 88 (1968), 492-517.

18. J-P. Serre, Corps locaux, Hermann, Paris, 1968.

19. J. Tate, Algorithm for determining the type of a singular fiber in an elliptic pencil, Lecture Notes in Math., vol. 476, Springer-Verlag, Berlin and New York, 1975, pp. 33-52.

20. H. Weber, Lehrbuch der algebra, vol. 3, Vieweg \& Sohn, Braunschweig, 1908.

21. A. Weil, Jacobi sums as 'Grössencharaktere', Trans. Amer. Math. Soc. 73 (1952), 487-495 (= Collected Papers, vol. II, Springer-Verlag, Berlin and New York, 1980, pp. 63-71).

Department of Mathematics, University of Georgia, Athens, Georgia 30602 